\title{
Prescribed Performance Neural Control of Strict-Feedback Systems via Disturbance Observers
}

\author{
Wei Xiang $(\mathbb{D}$, Guangkui Xu, Fang Zhu, and Chunzhi Yang \\ Department of Applied Mathematics, Huainan Normal University, Huainan 232038, China \\ Correspondence should be addressed to Wei Xiang; xiangwei27@126.com
}

Received 20 September 2020; Revised 11 October 2020; Accepted 15 October 2020; Published 29 October 2020

Academic Editor: Ahmed Mostafa Khalil

Copyright (@) 2020 Wei Xiang et al. This is an open access article distributed under the Creative Commons Attribution License, which permits unrestricted use, distribution, and reproduction in any medium, provided the original work is properly cited.

\begin{abstract}
This paper provides a disturbance observer-based prescribed performance control method for uncertain strict-feedback systems. To guarantee that the tracking error meets a design prescribed performance boundary (PPB) condition, an improved prescribed performance function is introduced. And radial basis function neural networks (RBFNNs) are used to approximate nonlinear functions, while second-order filters are employed to eliminate the "explosion-complexity" problem inherent in the existing method. Meanwhile, disturbance observers are constructed to estimate the compounded disturbance which includes time-varying disturbances and network construction errors. The stability of the whole closed-loop system is guaranteed via Lyapunov theory. Finally, comparative simulation results confirm that the proposed control method can achieve better tracking performance.
\end{abstract}

\section{Introduction}

Backstepping technology is widely used for nonlinear systems with the strict-feedback structure [1-3]. This method requires repeated differentiation of the virtual controller, which leads to the problem of "explosion-complexity." In order to solve this problem and estimate the virtual controller, dynamic surface control (DSC) technique [4-7] is applied. In [4], DSC technology was applied to the state-feedback adaptive control law, which eliminated the problem of "explosion-complexity" and ensured that all signals in the closed-loop system were uniformly ultimately bounded. By using DCS technology and fuzzy logic systems, Aung et al. [7] proposed a fuzzy adaptive control scheme that can guarantee tracking error to a small residual set. To further estimate the derivative of the virtual controller $\alpha(t)$, a famous first-order Levant differentiator was constructed in [8]. By applying the same differentiator, Yu et al. [9] proposed a backstepping approach, which enables the tracking error to converge to a small neighborhood in finite time. It can be seen from the above literature that the nonlinear system contains unknown nonlinear function terms and external disturbances. For the former, there are two approximate methods: one is using the composite learning control method [10-13] which uses online recorded data to produce a prediction error to construct a composite learning signal, and the other is using neural networks (NNs) or fuzzy logic systems (FLSs) [14-19]. However, adaptive laws of estimating parameters in [14-19] only consider whether the Lyapunov stability condition is satisfied but does not consider whether the parameter law can accurately estimate the unknown nonlinear function itself. For the latter, we cannot just assume that the external disturbance is bounded and the upper bound is unknown. And this assumption will lead to a problem where the disturbance cannot be accurately estimated. Currently, the effective method is to build a disturbance observer. The basic principle is that all disturbances are regarded as system states. In [20], a neural network disturbance observer (NNDOB) was developed for a 3-DOF model helicopter system. In [21], disturbance observerbased composite control was proposed for the flexible-link manipulator. In order to estimate unknown system nonlinearity and unknown disturbance, composite learning control strategy for uncertain systems in strict-feedback form was proposed in $[22,23]$. Some other interesting results about disturbance observer-based control can be seen in [24-27].

On the contrary, especially in practical control, it is better to specify a prescribed performance boundary (PPB) 
of tracking error rather than the approach tracking error to a small neighborhood. Therefore, how to combine the prescribed performance control (PPC) $[28,29]$ and the disturbance observer to make the error meet the PPB condition has become a research focus. Usually, the traditional PPB [30] of tracking error $e$ is described as

$$
\begin{aligned}
-\lambda \mu(t) & <e<\mu(t) \text { if } e(0)>0, \\
-\mu(t) & <e<\lambda \mu(t) \text { if } e(0)>0,
\end{aligned}
$$

where $\lambda \in[0,1]$ and $\mu(t)=\left(\mu_{0}-\mu_{\infty}\right) e^{-\lambda_{1} t}+\mu_{\infty}$; $\mu_{0}, \mu_{\infty}$, and $\lambda_{1}$ are design parameters. However, the traditional PPC strategy has two shortcomings. One is that the design of the controller needs to consider the sign of the initial value $e(0)$, and the other is that the small convergence overshoot of $e$ cannot be guaranteed. To tackle the above defects, an improved PPC methodology was proposed in [31]. And Bu et al. [31] only discussed the situation that external disturbances were unknown. However, some known information of nonlinear functions may not be able to provide in the practical control system. Therefore, it is necessary to discuss that how to accurately estimate unknown nonlinear functions and external disturbances under the condition that the tracking error meets the PPB.

Inspired by the above discussion, a class of SISO uncertain nonlinear system in strict-feedback form with unknown time-varying disturbance will be studied in this paper. Different from previous designs, an improved prescribed performance control scheme is proposed. The overall framework of this paper is as follows: firstly, a specific performance function and PPB condition are given. Then, the original system is transformed into an equivalent transformation system. Afterwards, unknown nonlinear functions are approximated by using RBFNNs, and a disturbance observer is designed to estimate the compounded disturbance which includes time-varying disturbances and network construction errors. The proposed composite prescribed performance control scheme can not only guarantee the prescribed performance but also make accurate estimates of system uncertainties. The main contributions of this paper are summarized as follows: (1) the proposed PPC method guarantees a small convergence overshoot of the tracking error; (2) system uncertainties (include unknown nonlinear functions and external disturbances) can be effectively estimated by the disturbance observer and RBFNNs; and (3) compared with the traditional PPC method, the proposed method has better control performance. The remainder of this paper is organized as follows: Section 2 lists some preliminaries. In Section 3, a novel PPC scheme is designed, and the stability of the closed-loop system is considered. Simulation studies are included in Section 4. Finally, brief conclusion is presented in Section 5.

\section{Preliminaries}

2.1. System Description. Consider the following SISO strictfeedback nonlinear system:

$$
\left\{\begin{array}{l}
\dot{x}_{1}=f_{1}\left(\overline{\mathbf{x}}_{1}\right)+g_{1}\left(\overline{\mathbf{x}}_{1}\right) x_{2}+d_{1}(t), \\
\dot{x}_{2}=f_{2}\left(\overline{\mathbf{x}}_{2}\right)+g_{1}\left(\overline{\mathbf{x}}_{2}\right) x_{3}+d_{2}(t), \\
\cdots \\
\dot{x}_{n}=f_{n}\left(\overline{\mathbf{x}}_{n}\right)+g_{n}\left(\overline{\mathbf{x}}_{n}\right) u+d_{n}(t), \\
y=x_{1},
\end{array}\right.
$$

where $\quad \overline{\mathbf{x}}_{i}=\left[x_{1}, x_{2}, \ldots, x_{i}\right]^{T} \in R^{i}, \quad x_{1}=\overline{\mathbf{x}}_{1} \in R, \quad \mathbf{x}=\overline{\mathbf{x}}_{n}=$ $\left[x_{1}, x_{2}, \ldots, x_{n}\right]^{T} \in R^{n}, u \in R$ is the control input, $y \in R$ is the output, $f_{i}\left(\overline{\mathbf{x}}_{i}\right)$ is an unknown smooth function, $g_{i}\left(\overline{\mathbf{x}}_{i}\right)$ is a known nonzero function, and $d_{i}(t)$ is the external timevarying disturbance, $i=1,2, \ldots, n$.

Assumption 1. The reference signal $x_{d}$ and its derivative $\dot{x}_{d}$ are available and bounded.

Assumption 2. There exist positive constants $\bar{d}_{i}, d_{i}^{*}$, and $\bar{g}_{i}$ such that $\left|d_{i}(t)\right| \leq \bar{d}_{i}, \quad\left|\dot{d}_{i}(t)\right| \leq d_{i}^{*}$, and $\left|g_{i}\left(\bar{x}_{i}\right)\right| \leq \bar{g}_{i}$, $i=1,2, \cdots, n$.

Remark 1. Assumptions 1 and 2 are common assumptions for strict-feedback systems $[4,21,22]$. Here, the boundedness of the external disturbance and its derivative is to construct the disturbance observer, and positive constants $\bar{d}_{i}, d_{i}^{*}$, and $\bar{g}_{i}$ are only used for stability analysis.

For SISO strict-feedback nonlinear system (2), backstepping technique will be used in the design process of controller $u$, and the tedious derivative calculation of the virtual control law will cause the problem of "explosion of terms." To avoid such problems, the following second-order filters are used in this paper.

Lemma 1 (see [28]). Consider the following system:

$$
\left\{\begin{array}{l}
\dot{\chi}_{1}=\chi_{2}, \\
\dot{\chi}_{2}=\oplus_{1}^{2}\left[-\oplus_{2} \tanh \left(\chi_{1}-\xi\right)-\oplus_{3} \tanh \left(\frac{\chi_{2}}{\varpi_{1}}\right)\right],
\end{array}\right.
$$

where $\Phi_{1}, \Phi_{2}$, and $\Phi_{3}$ are design parameters and $\xi$ is the input signal. From [28], it can be concluded that there exists a positive constant $\varrho$ such that $\chi_{i}-\xi^{(i-1)}=O\left(\left(1 / \varpi_{1}\right)^{(\varrho \omega-i+1)}\right)$, $i=1,2$, where $\omega=((1-\bar{\omega}) / \bar{\omega})$ and $\bar{\omega} \in \min \{\varrho /(1+\varrho), 1 / 2\}$. Therefore, there exist sufficiently small positive constants $\kappa_{1}$ and $\kappa_{2}$ such that $\left|\epsilon_{1}\right| \leq \kappa_{1}$ and $\left|\epsilon_{2}\right| \leq \kappa_{2}$, where $\epsilon_{1}=\chi_{1}-\xi$ and $\epsilon_{2}=\chi_{2}-\dot{\xi}$.

In order to estimate the function $f_{i}\left(\overline{\mathbf{x}}_{i}\right)$, we define $F_{i}\left(\overline{\mathbf{x}}_{i}\right)=l_{i} f_{i}\left(\overline{\mathbf{x}}_{i}\right) ; l_{i}$ is the positive design constant. According to the approximation property of RBFNNs, the approximation form of $F_{i}\left(\overline{\mathbf{x}}_{i}\right)$ can be expressed as

$$
\widehat{F}_{i}\left(\overline{\mathbf{x}}_{i}\right)=\widehat{\theta}_{F_{i}}^{T} \varphi_{F_{i}}\left(\overline{\mathbf{x}}_{i}\right),
$$

where $\widehat{\theta}_{F_{i}}=\left[\widehat{\theta}_{F_{i 1}}, \widehat{\theta}_{F_{i 2}}, \ldots, \widehat{\theta}_{F_{i s}}{ }^{T} \in R^{s}\right.$ is the weight vector, $s$ is the node number, and $\varphi_{F_{i}}\left(\overline{\mathbf{x}}_{i}\right)=\left[\varphi_{F_{i 1}}\left(\overline{\mathbf{x}}_{i}\right), \varphi_{F_{i 2}}\left(\overline{\mathbf{x}}_{i}\right), \ldots\right.$, $\left.\varphi_{F_{i s}}\left(\overline{\mathbf{x}}_{i}\right)\right]^{T} \in R^{s}$ is the basis function vector. Here, $\varphi_{F_{i j}}\left(\overline{\mathbf{x}}_{i}\right), j=1,2, \ldots, s$, is 


$$
\varphi_{F_{i j}}\left(\overline{\mathbf{x}}_{i}\right)=\exp \left(\frac{-\left(\overline{\mathbf{x}}_{i}-\mathbf{c}_{j}\right)^{T}\left(\overline{\mathbf{x}}_{i}-\mathbf{c}_{j}\right)}{2 b_{j}^{2}}\right),
$$

where $\mathbf{c}_{j}=\left[c_{j 1}, c_{j 2}, \ldots, c_{j i}\right]^{T} \in R^{i}$ and $b_{j}$ are the center vector and width of the basis function, respectively.

By [2-4], choosing appropriate $\mathbf{c}_{j}$ and $b_{j}$ and some sufficiently large integer $s$, there exists an ideal weight vector $\theta_{F_{i}}^{*} \in R^{s}$ such that

$$
F_{i}\left(\overline{\mathbf{x}}_{i}\right)=\theta_{F_{i}}^{* T} \varphi_{F_{i}}\left(\overline{\mathbf{x}}_{i}\right)+\varepsilon_{F_{i}},
$$

where $\varepsilon_{F_{i}}$ is the approximate error. So, we can rewrite $F_{i}\left(\overline{\mathbf{x}}_{i}\right)$ in (6) as

$$
F_{i}\left(\overline{\mathbf{x}}_{i}\right)=\tilde{\theta}_{F_{i}}^{T} \varphi_{F_{i}}\left(\overline{\mathbf{x}}_{i}\right)+\hat{\theta}_{F_{i}}^{T} \varphi_{F_{i}}\left(\overline{\mathbf{x}}_{i}\right)+\varepsilon_{F_{i}},
$$

where $\widetilde{\theta}_{F_{i}}=\theta_{F_{i}}^{*}-\widehat{\theta}_{F_{i}}$, and $\widehat{\theta}_{F_{i}}$ is the estimation of $\theta_{F_{i}}^{*}$.

Notice that $F_{i}\left(\overline{\mathbf{x}}_{i}\right)=l_{i} f_{i}\left(\overline{\mathbf{x}}_{i}\right)$, so we have

$$
f_{i}\left(\overline{\mathbf{x}}_{i}\right)=l_{i}^{-1} F_{i}\left(\overline{\mathbf{x}}_{i}\right)=l_{i}^{-1} \widetilde{\theta}_{F_{i}}^{T} \varphi_{F_{i}}\left(\overline{\mathbf{x}}_{i}\right)+l_{i}^{-1} \widehat{\theta}_{F_{i}}^{T} \varphi_{F_{i}}\left(\overline{\mathbf{x}}_{i}\right)+l_{i}^{-1} \varepsilon_{F_{i}} .
$$

Substituting (8) into (2), we get

$$
\begin{aligned}
\dot{x}_{1} & =l_{1}^{-1} \widehat{\theta}_{F_{1}}^{T} \varphi_{F_{1}}\left(\overline{\mathbf{x}}_{1}\right)+l_{1}^{-1} \widetilde{\theta}_{F_{1}}^{T} \varphi_{F_{1}}\left(\overline{\mathbf{x}}_{1}\right)+g_{1}\left(\overline{\mathbf{x}}_{1}\right) x_{2}+D_{1}, \\
\dot{x}_{2} & =l_{2}^{-1} \widehat{\theta}_{F_{2}}^{T} \varphi_{F_{2}}\left(\overline{\mathbf{x}}_{2}\right)+l_{2}^{-1} \widetilde{\theta}_{F_{2}}^{T} \varphi_{F_{2}}\left(\overline{\mathbf{x}}_{2}\right)+g_{2}\left(\overline{\mathbf{x}}_{2}\right) x_{3}+D_{2}, \\
& \ldots \\
\dot{x}_{n} & =l_{n}^{-1} \widehat{\theta}_{F_{n}}^{T} \varphi_{F_{n}}\left(\overline{\mathbf{x}}_{n}\right)+l_{n}^{-1} \widetilde{\theta}_{F_{n}}^{T} \varphi_{F_{n}}\left(\overline{\mathbf{x}}_{n}\right)+g_{n}\left(\overline{\mathbf{x}}_{n}\right) u+D_{n},
\end{aligned}
$$

where $D_{i}=d_{i}(t)+l_{i}^{-1} \varepsilon_{F_{i}}, i=1,2, \ldots, n$.

Remark 2. From (9), the design parameters $l_{1}, l_{2}, \ldots, l_{n}$ need to meet certain conditions, which will be given in the subsequent stability analysis. In [31], $\mathrm{Bu}$ et al. did not consider the case that nonlinear functions $f_{i}\left(\overline{\mathbf{x}}_{i}\right), i=1,2, \ldots, n$, are unknown and how to estimate them accurately. Therefore, the results of this paper can be regarded as a further study of [31]. For parameters $l_{1}, l_{2}, \ldots, l_{n}$ in (9), the stability conditions of them will be given in the later analytical proof.

2.2. Prescribed Performance. Define the tracking error variable $e_{1}=x_{1}-x_{d}$, and set $e$ satisfies the following prescribed performance boundary (PPB) [31]:

$$
p_{1}(t)<e_{1}<p_{2}(t),
$$

where $p_{1}(t)$ and $p_{2}(t)$ are performance functions and defined as

$$
\begin{aligned}
p_{1}(t) & =\left[\operatorname{sign}\left(e_{1}(0)\right)-\lambda_{1}\right] \mu(t)-\mu_{\infty} \operatorname{sign}\left(e_{1}(0)\right), \\
p_{2}(t) & =\left[\operatorname{sign}\left(e_{1}(0)\right)+\lambda_{2}\right] \mu(t)-\mu_{\infty} \operatorname{sign}\left(e_{1}(0)\right), \\
\mu(t) & =\left(\mu_{0}-\mu_{\infty}\right) e^{-\lambda_{3} t}+\mu_{\infty},
\end{aligned}
$$

where $0 \leq \lambda_{1}, \lambda_{2} \leq 1, \mu_{0}>\mu_{\infty}>0$, and $\lambda_{3}>0$.
In order to ensure that the tracking error $e$ is limited within PPB (10), we introduce the following transformation variable $z_{1}$ :

$$
z_{1}=\ln \left(\frac{h(t)}{1-h(t)}\right)
$$

where $h(t)=\left(e_{1}(t)-p_{1}(t) / p_{2}(t)-p_{1}(t)\right)$.

Lemma 2. If $z_{1}$ is bounded, $e_{1}$ can be limited within $P P B$ (10).

Proof. Since $z_{1}$ is bounded, there exists a constant $M>0$ such that $\left|z_{1}\right| \leq M$. Notice $z_{1}=\ln (h(t) / 1-h(t))$ is equivalent to $h(t)=\left(e^{z_{1}} / 1+e^{z_{1}}\right)$. So,

$$
0<\frac{e^{-M_{1}}}{1+e^{-M_{1}}}<h(t)=\frac{e_{1}(t)-p_{1}(t)}{p_{2}(t)-p_{1}(t)}<\frac{e^{M_{1}}}{1+e^{M_{1}}}<1 .
$$

Clearly, we have $p_{1}(t)<e_{1}(t)<p_{2}(t)$. The proof is completed.

Remark 3. Compared with [30], prescribed performance function (11) in this paper can guarantee the convergence of $e_{1}$ in a small overshoot, which is depicted in Figure 1.

When the tracking error $e_{1}$ is limited within PPB (10) by choosing appropriate parameters $\mu_{0}, \mu_{\infty}, \lambda_{1}, \lambda_{2}$, and $\lambda_{3}$, we can conclude that (i) $r_{1}=\left(1 / h(t)(1-h(t))\left(p_{2}(t)-\right.\right.$ $\left.\left.p_{1}(t)\right)\right)>0$; (ii) $r_{1}$ tends to a small neighborhood of a certain constant, and then $\dot{r}_{1}$ also tends to a small neighborhood of zero. Therefore, based on the above two properties of $r_{1}$ and Lemma 2 , we use $\left(1 / 2 r_{1}\right) z_{1}^{2}$ instead of $(1 / 2) z_{1}^{2}$ to explore the boundedness of $z_{1}$.

2.3. Control Aim. The aim of this paper is (i) to design the composite prescribed performance control scheme so that the tracking error $e_{1}$ satisfies PPB (10) and (ii) to construct the disturbance observer $\widehat{D}_{i}$ and neural network rules (NNs) so that $f_{i}\left(\overline{\mathbf{x}}_{i}\right)+d_{i}(t)$ can be estimated accurately.

Remark 4. It is necessary to point out that the disturbance observer $\widehat{D}_{i}$ in this paper is not to estimate the external disturbance $d_{i}(t)$ but to estimate accurately the external disturbance $d_{i}(t)$ and unknown function $f_{i}\left(\overline{\mathbf{x}}_{i}\right), i=$ $1,2, \ldots, n$.

\section{Control Design}

In order to prove that $z_{1}$ is bounded, the prescribed performance control and backstepping technique will be employed in this section. And the controller $u$ will be designed at step $n$.

Step 1. The time derivative of $z_{1}$ becomes

$$
\dot{z}_{1}=r_{1}\left(l_{1}^{-1} \widetilde{\theta}_{F_{1}}^{T} \varphi_{F_{1}}\left(\overline{\mathbf{x}}_{1}\right)+l_{1}^{-1} \widehat{\theta}_{F_{1}}^{T} \varphi_{F_{1}}\left(\overline{\mathbf{x}}_{1}\right)+g_{1}\left(\overline{\mathbf{x}}_{1}\right) x_{2}+D_{1}-\dot{x}_{d}+\Pi\right),
$$




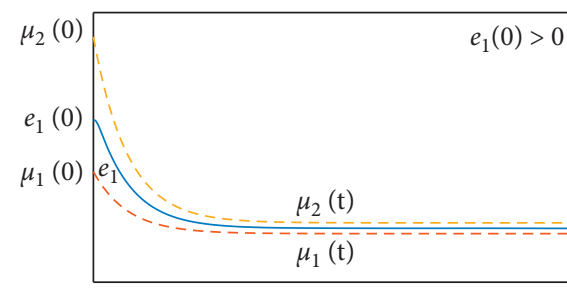

Time (second)

(a)

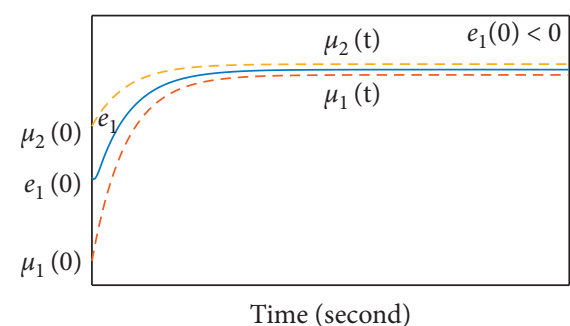

(b)

FIGURE 1: Graphical illustration of prescribed performance function (11) with (a) $e_{1}(0)>0$; (b) $e_{1}(0)<0$.

where

$$
\begin{aligned}
& r_{1}=\frac{1}{h(t)(1-h(t))\left(p_{2}(t)-p_{1}(t)\right)}>0, \\
& \Pi=\frac{p_{1}(t) \dot{p}_{2}(t)-\dot{p}_{1}(t) p_{2}(t)-e_{1}\left(\dot{p}_{2}(t)-p_{1}(t)\right)}{p_{2}(t)-p_{1}(t)} .
\end{aligned}
$$

Remark 5. According to (10) and (12), we know that $p_{2}(t)-p_{1}(t)>0, \quad 1>h(t)>0, \quad$ and $\quad 1-h(t)>0, \quad$ so $r_{1}=\left(1 / h(t)(1-h(t))\left(p_{2}(t)-p_{1}(t)\right)\right)>0$.

Choose virtual control $\alpha_{1}$ as

$$
\alpha_{1}=\frac{-l_{1}^{-1} \widehat{\theta}_{F_{1}}^{T} \varphi_{F_{1}}\left(\overline{\mathbf{x}}_{1}\right)-\widehat{D}_{1}+\dot{x}_{d}-\Pi-c_{1} z_{1}}{g_{1}\left(\overline{\mathbf{x}}_{1}\right)}
$$

where $\widehat{D}_{1}$ is the estimation of $D_{1}$ and $c_{1}$ is a positive design constant.

Define $z_{2}=x_{2}-\xi_{21}$, where $\xi_{21}$ is used to estimate $\alpha_{1}$ through the following second-order filter:

$$
\begin{aligned}
& \dot{\xi}_{21}=\xi_{22}, \\
& \dot{\xi}_{22}=\lambda_{21}^{2}\left[-\lambda_{22} \tanh \left(\xi_{21}-\alpha_{1}\right)-\lambda_{23} \tanh \left(\frac{\xi_{22}}{\lambda_{21}}\right)\right],
\end{aligned}
$$

where $\lambda_{21}, \lambda_{22}$, and $\lambda_{23}$ are design parameters. By Lemma 1, we know that there exists a positive constant $M_{1}$ such that $\left|\xi_{21}-\alpha_{1}\right| \leq M_{1}$. Define $e_{2}=\xi_{21}-\alpha_{1}$ and a prediction error $\widetilde{z}_{1}$ as $\widetilde{z}_{1}=\widehat{z}_{1}-z_{1}$, where $\widehat{z}_{1}$ satisfies

$$
\dot{\vec{z}}_{1}=r_{1}\left(l_{1}^{-1} \widehat{\theta}_{F_{1}}^{T} \varphi_{F_{1}}\left(\overline{\mathbf{x}}_{1}\right)+g_{1}\left(\overline{\mathbf{x}}_{1}\right) x_{2}+\widehat{D}_{1}-\dot{x}_{d}+\Pi-k_{1} \widetilde{z}_{1}\right),
$$

where $k_{1}$ is a positive design constant. The disturbance observer is designed as

$$
\begin{aligned}
\widehat{D}_{1}= & l_{01}\left(z_{1}-\omega_{1}\right), \\
\dot{\omega}_{1}= & r_{1}\left(l_{1}^{-1} \widehat{\theta}_{F_{1}}^{T} \varphi_{F_{1}}\left(\overline{\mathbf{x}}_{1}\right)+g_{1}\left(\overline{\mathbf{x}}_{1}\right) x_{2}+\widehat{D}_{1}-\dot{x}_{d}+\Pi\right. \\
& \left.+l_{01}^{-1}\left(\gamma_{1} \widetilde{z}_{1}-z_{1}\right)\right),
\end{aligned}
$$

where $l_{01}$ and $\gamma_{1}$ are positive constants. It should be pointed out that the purpose of introducing the prediction error here is to provide the information of the error state so that the following parameter adaptive law can ensure that the NN effectively estimates the unknown function.

The composite parameter adaptive law for $\widehat{\theta}_{F_{1}}$ is designed as

$$
\dot{\hat{\theta}}_{F_{1}}=\lambda_{F_{1}}\left(l_{1}^{-1} z_{1} \varphi_{F_{1}}\left(\overline{\mathbf{x}}_{1}\right)-\gamma_{1} l_{1}^{-1} \widetilde{z}_{1} \varphi_{F_{1}}\left(\overline{\mathbf{x}}_{1}\right)-\delta_{1} \widehat{\theta}_{F_{1}}\right),
$$

where $\lambda_{F_{1}}$ and $\delta_{1}$ are positive design parameters.

Define the following Lyapunov function:

$$
V_{1}=\frac{1}{2 r_{1}}\left(z_{1}^{2}+\gamma_{1} \widetilde{z}_{1}^{2}+\widetilde{D}_{1}^{2}\right)+\frac{1}{2 \lambda_{F_{1}}} \tilde{\theta}_{F_{1}}^{T} \tilde{\theta}_{F_{1}},
$$

where $\widetilde{D}_{1}=D_{1}-\widehat{D}_{1}$. According to (14)-(20) and $x_{2}=z_{2}+e_{2}+\alpha_{1}$, the time derivative of $V_{1}$ is

$$
\begin{aligned}
\dot{V}_{1}= & -\left(c_{1}+\frac{\dot{r}_{1}}{2 r_{1}^{2}}\right) z_{1}^{2}-\gamma_{1}\left(k_{1}+\frac{\dot{r}_{1}}{2 r_{1}^{2}}\right) \widetilde{z}_{1}^{2}+z_{1} g_{1}\left(\overline{\mathbf{x}}_{1}\right) z_{2} \\
& +z_{1} g_{1}\left(\overline{\mathbf{x}}_{1}\right) e_{2} \\
& -\left(l_{01}+\frac{\dot{r}_{1}}{2 r_{1}^{2}}\right) \widetilde{D}_{1}^{2}+\frac{\widetilde{D}_{1} \dot{D}_{1}}{r_{1}}-l_{01} l_{1}^{-1} \widetilde{D}_{1} \widetilde{\theta}_{F_{1}}^{T} \varphi_{F_{1}}\left(\overline{\mathbf{x}}_{1}\right) \\
& +\delta_{1} \widetilde{\theta}_{F_{1}}^{T} \widehat{\theta}_{F_{1}}^{T}
\end{aligned}
$$

If $z_{2}$ is bounded, i.e., there exists a positive constant $K_{2}$ such that $\left|z_{2}\right| \leq K_{2}$, and according to Young's inequality, we have

$$
\begin{gathered}
\frac{\widetilde{D}_{1} \dot{D}_{1}}{r_{1}} \leq \frac{\widetilde{D}_{1}}{2 r_{1}^{2}}+\frac{\dot{D}_{1}^{2}}{2} \leq \frac{\widetilde{D}_{1}}{2 r_{1}^{2}}+\frac{D_{1}^{* 2}}{2} \\
-l_{01} l_{1}^{-1} \widetilde{D}_{1} \widetilde{\theta}_{F_{1}}^{T} \varphi_{F_{1}}\left(\overline{\mathbf{x}}_{1}\right) \leq \frac{l_{01} l_{1}^{-1} \widetilde{D}_{1}^{2}}{2}+\frac{l_{01} l_{1}^{-1} \chi_{F_{1}}^{2}}{2}\left\|\widetilde{\theta}_{F_{1}}\right\|^{2}, \\
\delta_{1} \widetilde{\theta}_{F_{1}}^{T} \widehat{\theta}_{F_{1}} \leq-\frac{\delta_{1}}{2}\left\|\widetilde{\theta}_{F_{1}}\right\|^{2}+\frac{\delta_{1}}{2}\left\|\widetilde{\theta}_{F_{1}}^{*}\right\|^{2}, \\
z_{1} e_{2} g_{1}\left(\overline{\mathbf{x}}_{1}\right) \leq \frac{z_{1}^{2}}{2}+\frac{\bar{g}_{1} M_{1}^{2}}{2} \\
z_{1} g_{1}\left(\overline{\mathbf{x}}_{1}\right) z_{2} \leq \frac{z_{1}^{2}}{2}+\frac{\bar{g}_{1}^{2} K_{2}^{2}}{2}
\end{gathered}
$$


where $\left\|\varphi_{F_{1}}\left(\overline{\mathbf{x}}_{1}\right)\right\| \leq \chi_{F_{1}}$. Here, the boundedness of $\varphi_{F_{1}}\left(\bar{x}_{1}\right)$ can be referred to in $[12,13]$. Substituting (23) into (22), we obtain

$$
\begin{aligned}
\dot{V}_{1} \leq & -\left(c_{1}-1+\frac{\dot{r}_{1}}{2 r_{1}^{2}}\right) z_{1}^{2}-\gamma_{1}\left(k_{1}+\frac{\dot{r}_{1}}{2 r_{1}^{2}}\right) \tilde{z}_{1}^{2} \\
& -\left(l_{01}-\frac{l_{01} l_{1}^{-1}}{2}+\frac{\dot{r}_{1}-1}{2 r_{1}^{2}}\right) \widetilde{D}_{1}^{2}-\left(\frac{\delta_{1}}{2}-\frac{l_{01} l_{1}^{-1} \chi_{F_{1}}^{2}}{2}\right) \underbrace{\left\|\widetilde{\theta}_{F_{1}}\right\|^{2}}_{\tilde{\theta}_{F_{1}}^{T} \widetilde{\theta}_{F_{1}}}+R_{1},
\end{aligned}
$$

where $R_{1}=\left(D_{1}^{* 2} / 2\right)+\left(\delta_{1} / 2\right)\left\|\theta_{F_{1}}^{*}\right\|^{2}+\left(\bar{g}_{1}^{2} K_{2}^{2} / 2\right)+\left(\bar{g}_{1} M_{1}^{2} / 2\right)$. Let $c_{1}>1-\left(\dot{r}_{1} / 2 r_{1}^{2}\right), k_{1}>-\left(\dot{r}_{1} / 2 r_{1}^{2}\right), l_{01}>\left(l_{01} l_{1}^{-1} / 2\right)-\left(\dot{r}_{1}-1 /\right.$ $\left.2 r_{1}^{2}\right)$, and $\left(\delta_{1} / 2\right)>\left(l_{01} l_{1}^{-1} \chi_{F_{1}}^{2} / 2\right)$, and define the following compact sets:

$$
\begin{aligned}
& \Omega_{z_{1}}=\left\{z_{1}|| z_{1} \mid \leq \sqrt{\frac{R_{1}}{c_{1}-1+\dot{r}_{1} / 2 r_{1}^{2}}}\right\}, \\
& \Omega_{\widetilde{z}_{1}}=\left\{\widetilde{z}_{1}|| z_{1} \mid \leq \sqrt{\frac{R_{1}}{\gamma_{1}\left(k_{1}+\dot{r}_{1} / 2 r_{1}^{2}\right)}}\right\}, \\
& \Omega_{\widetilde{D}_{1}}=\left\{\widetilde{D}_{1}|| \widetilde{D}_{1} \mid \leq \sqrt{\frac{R_{1}}{l_{01}-l_{01} l_{1}^{-1} / 2+\dot{r}_{1}-1 / 2 r_{1}^{2}}}\right\}, \\
& \Omega_{\widetilde{\theta}_{F_{1}}}=\left\{\widetilde{\theta}_{F_{1}}|| \widetilde{\theta}_{F_{1}} \mid \leq \sqrt{\frac{R_{1}}{\delta_{1} / 2-l_{01} l_{1}^{-1} \chi_{F_{1}}^{2} / 2}}\right\} .
\end{aligned}
$$

It can be seen that $z_{1} \notin \Omega_{z_{1}}$ or $\widetilde{z}_{1} \notin \Omega_{\widetilde{z}_{1}}$ or $\widetilde{D}_{1} \notin \Omega_{\widetilde{D}_{1}}$ or $\widetilde{\theta}_{F_{1}} \notin \Omega_{\tilde{\theta}_{F_{1}}}$, and we have $\dot{V}_{1}<0$. Thus, $z_{1}, \widetilde{z}_{1}, \widetilde{D}_{1}$, and $\widetilde{\theta}_{F_{1}}$ are all semiglobally uniformly bounded. The above conclusion is based on the fact that the variable $z_{2}$ is bounded. Now, we need to prove that $z_{2}$ is bounded.

Step i. $i=2, \ldots, n-1$.

According to the definition of $z_{i}=x_{i}-\xi_{i 1}$, we have

$$
\dot{z}_{i}=l_{i}^{-1} \widetilde{\theta}_{F_{i}}^{T} \varphi_{F_{i}}\left(\overline{\mathbf{x}}_{i}\right)+l_{i}^{-1} \widehat{\theta}_{F_{i}}^{T} \varphi_{F_{i}}\left(\overline{\mathbf{x}}_{i}\right)+g_{i}\left(\overline{\mathbf{x}}_{i}\right) x_{i+1}+D_{i}-\xi_{i 2} .
$$

Choose virtual control $\alpha_{i}$ as

$$
\alpha_{i}=\frac{-l_{i}^{-1} \hat{\theta}_{F_{i}}^{T} \varphi_{F_{i}}\left(\overline{\mathbf{x}}_{i}\right)-\widehat{D}_{i}+\xi_{i 2}-c_{i} z_{i}}{g_{i}\left(\overline{\mathbf{x}}_{i}\right)},
$$

where $\widehat{D}_{i}$ is the estimation of $D_{i}$ and $c_{i}$ is a positive design constant.

Define $z_{i+1}=x_{i+1}-\xi_{i+1,1}$, and $\xi_{i+1,1}$ satisfies the following second-order filter:

$$
\begin{aligned}
& \dot{\xi}_{i+1,1}=\xi_{i+1,2}, \\
& \dot{\xi}_{i+1,2}=\lambda_{i+1,1}^{2}\left[-\lambda_{i+1,2} \tanh \left(\xi_{i+i, 1}-\alpha_{i}\right)-\lambda_{i+1,1} \tanh \left(\frac{\xi_{i+1,3}}{\lambda_{i+1,1}}\right)\right],
\end{aligned}
$$

where $\lambda_{i+1,1}, \lambda_{i+1,2}$, and $\lambda_{i+1,3}$ are design parameters. Define $e_{i+1}=\xi_{i+i, 1}-\alpha_{i}$, and there exists a positive constant $M_{i}$ such that $\left|e_{i+1}\right|=\left|\xi_{i+i, 1}-\alpha_{i}\right| \leq M_{i}$ by Lemma 1. Similar to Step 1, define the prediction variable $\widehat{z}_{i}$ as

$$
\dot{\bar{z}}_{i}=l_{i}^{-1} \widehat{\theta}_{F_{i}}^{T} \varphi_{F_{i}}\left(\overline{\mathbf{x}}_{i}\right)+g_{i}\left(\overline{\mathbf{x}}_{i}\right) x_{i+1}+\widehat{D}_{i}-\xi_{i 2}-k_{i} \widetilde{z}_{i},
$$

where $\widetilde{z}_{i}=\widehat{z}_{i}-z_{i}$ and $k_{i}>0$.

The disturbance observer is designed as

$$
\begin{aligned}
& \widehat{D}_{i}=l_{0 i}\left(z_{i}-w_{i}\right), \\
& \dot{w}_{i}=l_{i}^{-1} \widehat{\theta}_{F_{i}}^{T} \varphi_{F_{i}}\left(\overline{\mathbf{x}}_{i}\right)+g_{i}\left(\overline{\mathbf{x}}_{i}\right) x_{i+1}+\widehat{D}_{i}-\xi_{i 2}+l_{0 i}^{-1}\left(\gamma_{i} \widetilde{z}_{i}-z_{i}\right),
\end{aligned}
$$

where $l_{0 i}$ is a positive design parameter. And the composite parameter adaptive law for $\widehat{\theta}_{F_{i}}$ is designed as

$$
\dot{\hat{\theta}}_{F_{i}}=\lambda_{F_{i}}\left(l_{i}^{-1} z_{1} \varphi_{F_{i}}\left(\overline{\mathbf{x}}_{i}\right)-\gamma_{i} l_{i}^{-1} \widetilde{z}_{i} \varphi_{F_{i}}\left(\overline{\mathbf{x}}_{i}\right)-\delta_{i} \widehat{\theta}_{F_{i}}\right),
$$

where $\lambda_{F_{i}}$ and $\delta_{i}$ are positive design parameters.

Define the following Lyapunov function:

$$
V_{i}=\frac{1}{2}\left(z_{i}^{2}+\gamma_{i} \widetilde{z}_{i}^{2}+\widetilde{D}_{i}^{2}+\frac{1}{\lambda_{F_{i}}} \widetilde{\theta}_{F_{i}}^{T} \widetilde{\theta}_{F_{i}}\right),
$$

where $\widetilde{D}_{i}=D_{i}-\widehat{D}_{i}$.

According to (27)-(31) and $x_{i+1}=z_{i+1}+e_{i+1}+\alpha_{i}$, the time derivative of $V_{i}$ is

$$
\begin{aligned}
\dot{V}_{i}= & -c_{1} z_{1}^{2}-\gamma_{i} k_{i} \widetilde{z}_{i}^{2}+z_{i} g_{i}\left(\overline{\mathbf{x}}_{i}\right) z_{i+1}+z_{i} g_{i}\left(\overline{\mathbf{x}}_{i}\right) e_{i+1} \\
& -l_{0 i} \widetilde{D}_{1}^{2}+\widetilde{D}_{i} \dot{D}_{i}-l_{0 i} l_{i}^{-1} \widetilde{D}_{i} \widetilde{\theta}_{F_{i}}^{T} \varphi_{F_{i}}\left(\overline{\mathbf{x}}_{i}\right)+\delta_{i} \widetilde{\theta}_{F_{i}}^{T} \widehat{\theta}_{F_{i}}^{T} .
\end{aligned}
$$

If $z_{i+1}$ is bounded, i.e., $\left|z_{i+1}\right| \leq K_{i+1}, K_{i+1}$ is a positive constant, and according to Young's inequality, we have

$$
\widetilde{D}_{i} \dot{D}_{i} \leq \frac{\widetilde{D}_{i}}{2}+\frac{\dot{D}_{i}^{2}}{2} \leq \frac{\widetilde{D}_{i}}{2}+\frac{D_{i}^{* 2}}{2},
$$

$$
\begin{gathered}
-l_{0 i} l_{i}^{-1} \widetilde{D}_{i} \widetilde{\theta}_{F_{i}}^{T} \varphi_{F_{i}}\left(\overline{\mathbf{x}}_{i}\right) \leq \frac{l_{0 i} l_{i}^{-1} \widetilde{D}_{i}^{2}}{2}+\frac{l_{0 i} l_{i}^{-1} \chi_{F_{i}}^{2}}{2}\left\|\widetilde{\theta}_{F_{i}}\right\|^{2}, \\
\delta_{i} \widetilde{\theta}_{F_{i}}^{T} \widehat{\theta}_{F_{i}} \leq-\frac{\delta_{i}}{2}\left\|\widetilde{\theta}_{F_{i}}\right\|^{2}+\frac{\delta_{i}}{2}\left\|\widetilde{\theta}_{F_{i}}^{*}\right\|^{2}
\end{gathered}
$$

$$
\begin{aligned}
& z_{i} e_{i+1} g_{i}\left(\overline{\mathbf{x}}_{i}\right) \leq \frac{z_{i}^{2}}{2}+\frac{\bar{g}_{i} M_{i}^{2}}{2}, \\
& z_{i} g_{i}\left(\overline{\mathbf{x}}_{i}\right) z_{i+1} \leq \frac{z_{i}^{2}}{2}+\frac{\bar{g}_{i}^{2} K_{i+1}^{2}}{2},
\end{aligned}
$$

where $\left\|\varphi_{F_{i}}\left(\bar{x}_{i}\right)\right\| \leq \chi_{F_{i}}$. Substituting (34) into (33), we obtain

$$
\begin{aligned}
\dot{V}_{i} \leq & -\left(c_{i}-1\right) z_{i}^{2}-\gamma_{i} k_{i} \tilde{z}_{i}^{2}-\left(l_{0 i}-\frac{1}{2}-\frac{l_{0 i} l_{i}^{-1}}{2}\right) \widetilde{D}_{i}^{2} \\
& -\left(\frac{\delta_{i}}{2}-\frac{l_{0 i} l_{i}^{-1} \chi_{F_{i}}^{2}}{2}\right) \underbrace{\left\|\tilde{\theta}_{F_{i}}\right\|^{2}}_{\tilde{\theta}_{F_{i}} \widetilde{\theta}_{F_{i}}}+R_{i},
\end{aligned}
$$


where $R_{i}=\left(\gamma_{i}^{2} / 2\right)+\left(\delta_{i} / 2\right)\left\|\theta_{F_{i}}^{*}\right\|^{2}+\left(\bar{g}_{i}^{2} K_{i+1}^{2} / 2\right)+\left(\bar{g}_{i} M_{i}^{2} / 2\right)$. Let $c_{i}>1, \quad k_{i}>0, \quad l_{0 i}>\left(l_{0 i} l_{i}^{-1} / 2\right)+(1 / 2), \quad$ and $\quad\left(\delta_{i} / 2\right)>$ $\left(l_{0 i} i_{i}^{-1} \chi_{F_{i}}^{2} / 2\right)$, and define the following compact sets:

$$
\begin{aligned}
& \Omega_{z_{i}}=\left\{z_{i}|| z_{i} \mid \leq \sqrt{\frac{R_{i}}{c_{i}-1}}\right\}, \\
& \Omega_{\widetilde{z}_{i}}=\left\{\widetilde{z}_{i}|| \widetilde{z}_{i} \mid \leq \sqrt{\frac{R_{i}}{\gamma_{i} k_{i}}}\right\}, \\
& \Omega_{\widetilde{D}_{i}}=\left\{\widetilde{D}_{i}|| \widetilde{D}_{i} \mid \leq \sqrt{\frac{R_{i}}{l_{0 i}-\left(l_{0 i} l_{i}^{-1} / 2\right)-(1 / 2)}}\right\}, \\
& \Omega_{\widetilde{\theta}_{F_{i}}}=\left\{\widetilde{\theta}_{F_{i}}|| \widetilde{\theta}_{F_{i}} \mid \leq \sqrt{\frac{R_{i}}{\left(\delta_{i} / 2\right)-\left(l_{0 i} l_{i}^{-1} \chi_{F_{i}}^{2} / 2\right)}}\right\} .
\end{aligned}
$$

We know that if $z_{i} \notin \Omega_{z_{i}}$ or $\widetilde{z}_{i} \notin \Omega_{\tilde{z}_{i}}$ or $\widetilde{D}_{i} \notin \Omega \widetilde{D}_{\tilde{D}}$ or $\widetilde{\theta}_{F_{i}} \notin \Omega_{\theta_{\theta}}$, we have $\dot{V}_{i}<0$. Thus, $z_{i}, \widetilde{z}_{i}, \widetilde{D}_{i}$, and $\widetilde{\theta}_{F_{i}}$ are semiglobally uniformly bounded. So, to prove that $z_{i}$ is bounded, we have to prove that $z_{i+1}$ is bounded.

Step $n$. The time derivative of $z_{n}$ produces

$$
\dot{z}_{n}=l_{n}^{-1} \widehat{\theta}_{F_{n}}^{T} \varphi_{F_{n}}\left(\overline{\mathbf{x}}_{n}\right)+l_{n}^{-1} \widetilde{\theta}_{F_{n}}^{T} \varphi_{F_{n}}\left(\overline{\mathbf{x}}_{n}\right)+g_{n}\left(\overline{\mathbf{x}}_{n}\right) u+D_{n}-\xi_{n 2} .
$$

The control $u$ is designed as

$$
u=\frac{-l_{n}^{-1} \widehat{\theta}_{F_{n}}^{T} \varphi_{F_{n}}\left(\overline{\mathbf{x}}_{n}\right)-\widehat{D}_{n}+\xi_{n 2}-c_{n} z_{n}}{g_{n}\left(\overline{\mathbf{x}}_{n}\right)},
$$

where $\widehat{D}_{n}$ is the estimation of $D_{n}$ and $c_{n}$ is a positive constant. The prediction error $\widetilde{z}_{n}=\widehat{z}_{n}-z_{n}$, and $\widehat{z}_{n}$ satisfies

$$
\dot{\widehat{z}}_{n}=l_{n}^{-1} \widehat{\theta}_{F_{n}}^{T} \varphi_{F_{n}}\left(\overline{\mathbf{x}}_{n}\right)+g_{n}\left(\overline{\mathbf{x}}_{n}\right) u+\widehat{D}_{n}-\xi_{n 2}-k_{n} \widetilde{z}_{n} .
$$

The disturbance observer $\widehat{D}_{n}$ is designed as

$$
\begin{aligned}
& \widehat{D}_{n}=l_{0 n}\left(z_{n}-w_{n}\right), \\
& \dot{w}_{n}=l_{n}^{-1} \widehat{\theta}_{F_{n}}^{T} \varphi_{F_{n}}\left(\overline{\mathbf{x}}_{n}\right)+g_{n}\left(\overline{\mathbf{x}}_{n}\right) u+\widehat{D}_{n}-\xi_{n 2}+l_{0 n}^{-1}\left(\gamma_{n} \widetilde{z}_{n}-z_{n}\right),
\end{aligned}
$$

where $l_{0 n}$ is a positive design parameter. And the composite parameter adaptive law for $\widehat{\theta}_{F_{n}}$ is designed as

$$
\dot{\hat{\theta}}_{F_{n}}=\lambda_{F_{n}}\left[l_{n}^{-1} z_{n} \varphi_{F_{n}}\left(\overline{\mathbf{x}}_{n}\right)-\gamma_{n} l_{n}^{-1} \widetilde{z}_{n} \varphi_{F_{n}}\left(\overline{\mathbf{x}}_{n}\right)-\delta_{n} \widehat{\theta}_{F_{n}}\right],
$$

where $\lambda_{F_{n}}$ and $\delta_{n}$ are positive design parameters.

Consider the following Lyapunov function:

$$
V_{n}=\frac{1}{2}\left(z_{n}^{2}+\gamma_{n} \widetilde{z}_{n}^{2}+\widetilde{D}_{n}^{2}+\frac{1}{\lambda_{F_{n}}} \widetilde{\theta}_{F_{n}}^{T} \widetilde{\theta}_{F_{n}}\right),
$$

where $\widetilde{D}_{n}=D_{n}-\widehat{D}_{n}$.

According to (38)-(41), the time derivative of $V_{n}$ is

$$
\begin{aligned}
\dot{V}_{n}= & -c_{n} z_{n}^{2}-\gamma_{n} k_{n} \widetilde{z}_{n}^{2}-l_{0 n} \widetilde{D}_{n}^{2}+\widetilde{D}_{n} \dot{D}_{n} \\
& -l_{0 n} l_{n}^{-1} \widetilde{D}_{n} \widetilde{\theta}_{F_{n}}^{T} \varphi_{F_{n}}\left(\bar{x}_{n}\right)+\delta_{n} \widetilde{\theta}_{F_{n}}^{T} \widehat{\theta}_{F_{n}}^{T} .
\end{aligned}
$$

Consider the following facts:

$$
\begin{aligned}
\widetilde{D}_{n} \dot{D}_{n} \leq & \frac{\widetilde{D}_{n}}{2}+\frac{D_{n}^{* 2}}{2}, \\
& -l_{0 n} l_{n}^{-1} \widetilde{D}_{n} \widetilde{\theta}_{F_{n}}^{T} \varphi_{F_{n}}\left(\bar{x}_{n}\right)+\delta_{n} \widetilde{\theta}_{F_{n}}^{T} \widehat{\theta}_{F_{n}}^{T}, \\
\delta_{n} \widetilde{\theta}_{F_{n}}^{T} \widehat{\theta}_{F_{n}} \leq & -\frac{\delta_{n}}{2}\left\|\widetilde{\theta}_{F_{n}}\right\|^{2}+\frac{\delta_{n}}{2}\left\|\widetilde{\theta}_{F_{n}}^{*}\right\|^{2},
\end{aligned}
$$

where $\left\|\varphi_{F_{n}}\left(\bar{x}_{n}\right)\right\| \leq \chi_{F_{n}}$. Substituting (44) into (43), we obtain

$$
\begin{aligned}
\dot{V}_{n} \leq & -c_{n} z_{n}^{2}-\gamma_{n} k_{n} \widetilde{z}_{n}^{2}-\left(l_{0 n}-\frac{1}{2}-\frac{l_{0 n} l_{n}^{-1}}{2}\right) \widetilde{D}_{n}^{2} \\
& -\left(\frac{\delta_{n}}{2}-\frac{l_{0 n} l_{n}^{-1} \chi_{F_{n}}^{2}}{2}\right)\left\|\widetilde{\theta}_{F_{n}}\right\|^{2}+R_{n},
\end{aligned}
$$

where $R_{n}=\left(D_{n}^{* 2} / 2\right)+\left(\delta_{n} / 2\right)\left\|\theta_{F}^{*}\right\|^{2}$. Let $c_{n}>0, \quad k_{n}>0$, $l_{0 n}>\left(l_{0 n} l_{n}^{-1} / 2\right)+(1 / 2)$, and $\left(\delta_{n} / 2\right)>\left(l_{0 n} l_{n}^{-1} \chi_{F_{n}}^{2} / 2\right)$, and define the following compact sets:

$$
\begin{aligned}
& \Omega_{z_{n}}=\left\{z_{i}|| z_{n} \mid \leq \sqrt{\frac{R_{n}}{c_{n}}}\right\}, \\
& \Omega_{\widetilde{z}_{n}}=\left\{\widetilde{z}_{n}|| z_{n} \mid \leq \sqrt{\left.\frac{R_{n}}{\gamma_{n} k_{n}}\right\},}\right. \\
& \Omega_{\widetilde{D}_{n}}=\left\{\widetilde{D}_{n}|| \widetilde{D}_{n} \mid \leq \sqrt{\frac{R_{i}}{l_{0 n}-\left(l_{0 n} l_{n}^{-1} / 2\right)-(1 / 2)}}\right\}, \\
& \Omega_{\widetilde{\theta}_{F_{n}}}=\left\{\widetilde{\theta}_{F_{n}}|| \widetilde{\theta}_{F_{n}} \mid \leq \sqrt{\frac{R_{n}}{\left(\delta_{n} / 2\right)-\left(l_{0 n} l_{n}^{-1} \chi_{F_{n}}^{2} / 2\right)}}\right\} .
\end{aligned}
$$

Obviously, $\quad z_{n} \notin \Omega_{z_{n}}$ or $\quad \widetilde{z}_{n} \notin \Omega_{\widetilde{z}_{n}}$ or $\widetilde{D}_{n} \notin \Omega_{\widetilde{D}_{n}}$ or $\widetilde{\theta}_{F_{n}} \notin \Omega_{\tilde{\theta}_{F}}$, and we have $\dot{V}_{n}<0$. Thus, $z_{n}, \widetilde{z}_{n}, \widetilde{D}_{n}$, and $\widetilde{\theta}_{F_{n}}$ are semiglobally uniformly bounded. It can be seen that the controller $u$ can guarantee that $z_{n}$ is bounded, the boundedness of $z_{n}$ guarantees that $z_{n-1}$ is bounded, and the like, and $z_{2}$ guarantees that $z_{1}$ is bounded. So, we get $z_{1}$ is bounded in turn. According to Lemma 2, the tracking error $e_{1}$ satisfies PPB (10). We obtain the following main result.

Theorem 1. Consider strict-feedback nonlinear system (2) with uncertainties and unknown time-varying disturbances. If virtual controllers (16), (27), disturbance observers (19), (30), (40), the RBFNN updating laws (20), (31), (41), and real 


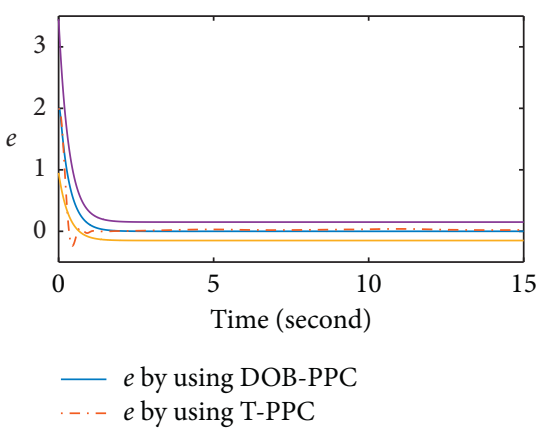

(a)

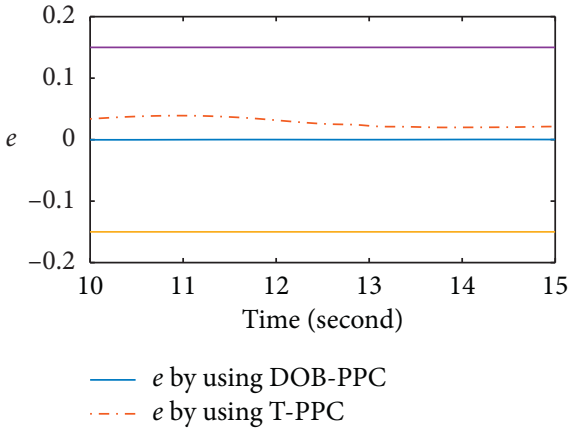

(b)

FIgURE 2: Tacking error $e$ of system (47) for two methods in time period (a) $[0,15 \mathrm{~s}]$; (b) [10 s, $15 \mathrm{~s}]$.

controller (37) are designed, it is guaranteed that the tracking error $e_{1}$ satisfies $P P B(10)$.

Remark 6. In this paper, neural network technology is adopted to transform system (2) into the equivalent of system (9). If the external disturbance $D_{i}=0$ in (9), it can be found from the construction of the disturbance observer that $l_{i}=0$ and $l_{0 i}=0, i=1,2, \cdots, n$. So, the control method in this paper can be applied to a wider range.

Remark 7. The traditional PPC control method can also be used in this paper, but the control effect is less than the proposed control method in this paper, which will be given intuitively through the figures in the simulations.

\section{Simulation Studies}

In the simulation, the following second-order strict-feedback nonlinear system is considered:

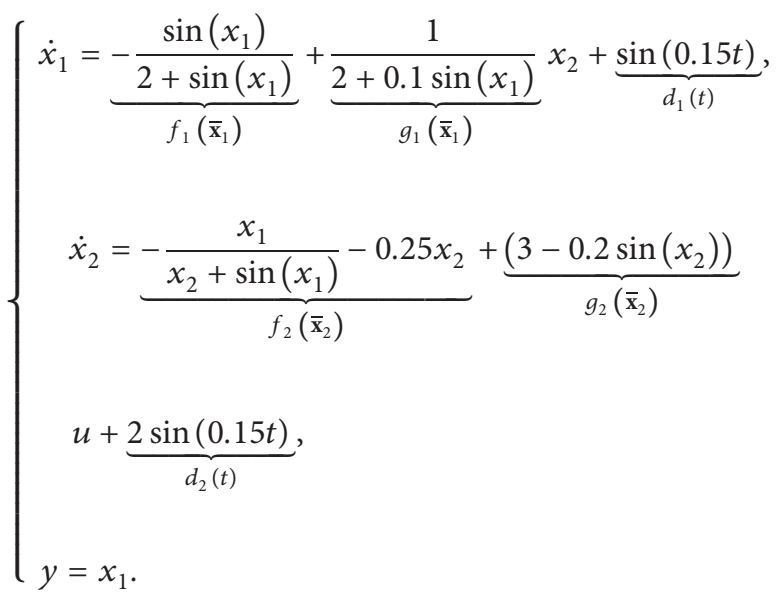

Denote the method in this paper as DOB-PPC. In order to compare with DOB-PPC, define $\bar{F}_{1}\left(\overline{\mathbf{x}}_{1}\right)=f_{1}\left(\overline{\mathbf{x}}_{1}\right)+d_{1}(t)$ and $\bar{F}_{2}\left(\overline{\mathbf{x}}_{2}\right)=f_{2}\left(\overline{\mathbf{x}}_{2}\right)+d_{2}(t)$, and the traditional PPC control method (denoted as T-PPC) is designed as

$$
\left\{\begin{array}{l}
u=\frac{-\widehat{\vartheta}_{\bar{F}_{2}}^{T} \varphi_{\bar{F}_{2}}\left(\overline{\mathbf{x}}_{2}\right)+\xi_{22}-c_{2} \bar{z}_{2}}{g_{2}\left(\overline{\mathbf{x}}_{2}\right)}, \\
\dot{\xi}_{21}=\xi_{22}, \\
\dot{\xi}_{22}=\lambda_{21}^{2}\left[-\lambda_{22} \tanh \left(\xi_{21}-\alpha_{1}\right)-\lambda_{23} \tanh \left(\frac{\xi_{22}}{\lambda_{21}}\right)\right], \\
\alpha_{1}=\frac{\widehat{\vartheta}_{\bar{F}_{1}}^{T} \varphi_{\bar{F}_{1}}\left(\overline{\mathbf{x}}_{1}\right)+\dot{x}_{d}-\bar{\Pi}-c_{1} \bar{z}_{1}}{g_{1}\left(\overline{\mathbf{x}}_{1}\right)} \\
\dot{\widehat{\vartheta}}_{\bar{F}_{1}}=\lambda_{\bar{F}_{1}}\left(\bar{z}_{1} \varphi_{\bar{F}_{1}}\left(\overline{\mathbf{x}}_{1}\right)-\delta_{1} \widehat{\vartheta}_{\bar{F}_{1}}\right), \\
\dot{\hat{\vartheta}}_{\bar{F}_{2}}=\lambda_{\bar{F}_{2}}\left(\bar{z}_{2} \varphi_{\bar{F}_{2}}\left(\overline{\mathbf{x}}_{2}\right)-\delta_{2} \widehat{\vartheta}_{\bar{F}_{2}}\right), \\
\bar{z}_{1}=\tanh \left(\frac{e}{\mu}\right), \\
\mu(2.5-0.3) \exp (-3.0 t)+0.3 .
\end{array}\right.
$$

The stability proof of T-PPC (48) is given in Appendix 1. It can be found that only the RBFNN is used to estimate system uncertainty in T-PPC (48). The parameters of T-PPC are chosen as $\lambda_{F_{1}}=\lambda_{F_{2}}=40, \quad c_{1}=c_{2}=5, \quad \lambda_{21}=150$, $\lambda_{22}=\lambda_{23}=5$, and $\delta_{1}=\delta_{2}=0.5$. $\quad N_{1}=17$ and $N_{2}=45$ ( $N_{1}$ and $N_{2}$ are the dimensions of $\widehat{\vartheta}_{F_{1}}$ and $\widehat{\vartheta}_{F_{2}}$, respectively). The centers of $\overline{\mathbf{x}}_{1}$ and $\overline{\mathbf{x}}_{2}$ are evenly spaced in $[0,8]$ and $[0,8] \times[-1,3]$, respectively.

For method of DOB-PPC, the above parameters are the same. In addition, select $l_{1}=l_{2}=10, l_{01}=l_{02}=20$, $\gamma_{1}=\gamma_{2}=3, k_{1}=k_{2}=5$, and $\lambda_{1}=\lambda_{2}=(1 / 2)$. The dimension of $\widehat{\theta}_{F_{1}}$ is $N_{1}=17$, and the dimension of $\hat{\theta}_{F_{2}}$ is $N_{2}=81$. The centers of $\overline{\mathbf{x}}_{1}$ and $\overline{\mathbf{x}}_{2}$ are the same as ones in the method of T-PPC. The initial value $\mathbf{x}(0)=\left[x_{1}(0), x_{2}(0)\right]^{T}=[2,2]^{T}$, and $x_{d}=\sin (t)$. Firstly, the tracking error effect of T-PPC and DOB-PPC is compared, and the result in Figure 2 


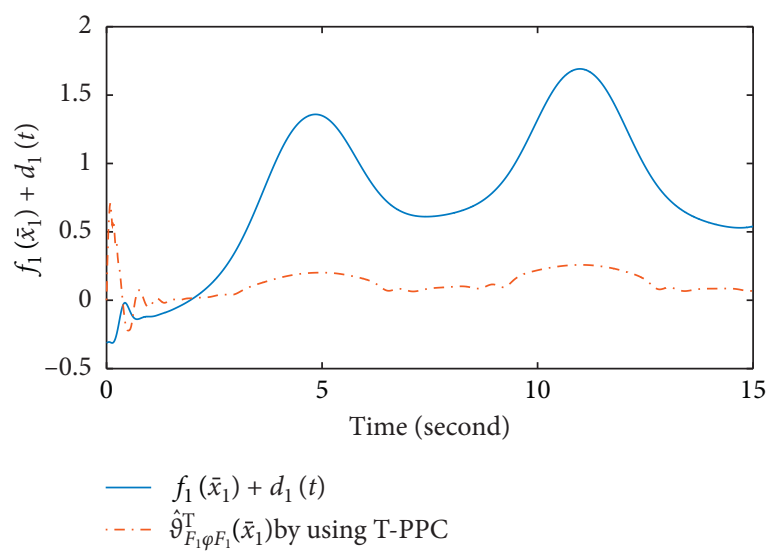

(a)

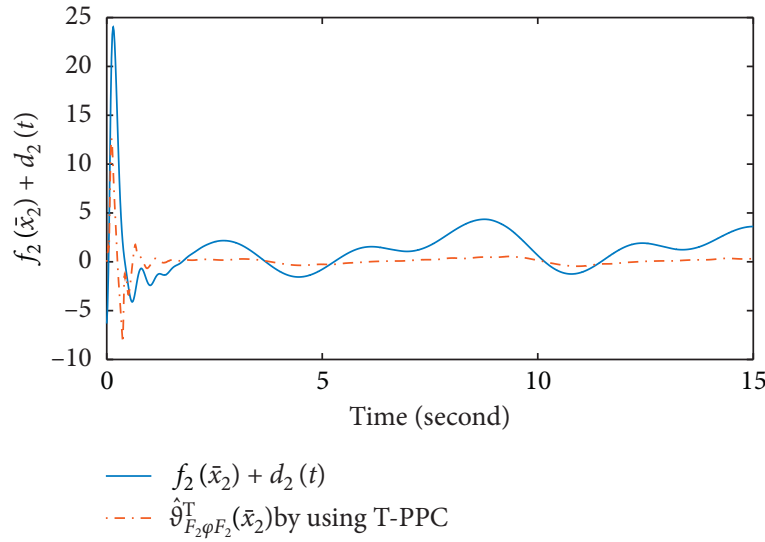

(c)

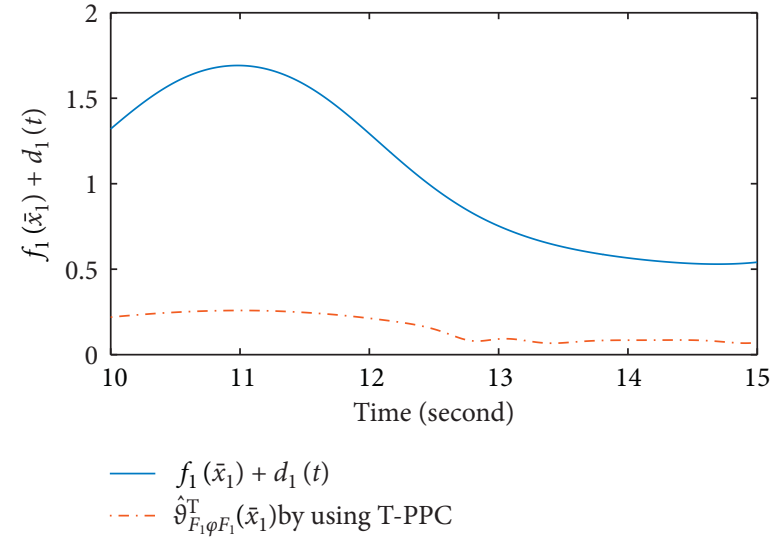

(b)

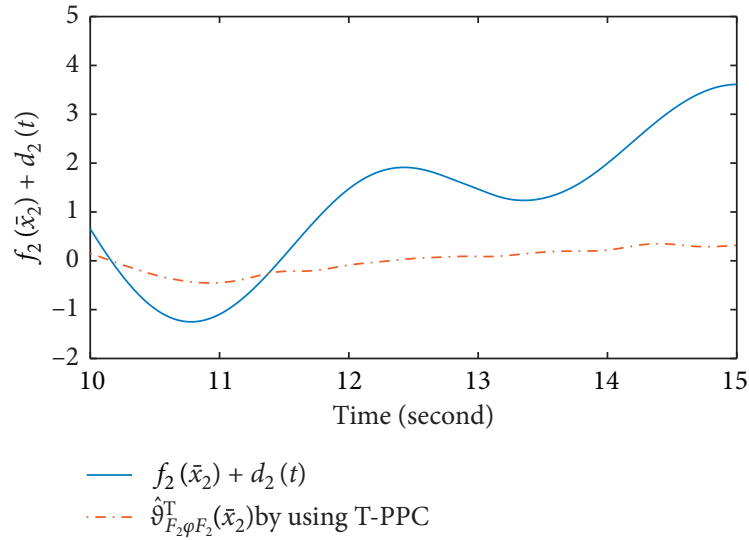

(d)

FIGURe 3: Estimation of $f_{1}\left(\bar{x}_{1}\right)+d_{1}(t)$ by using T-PPC in time period (a) [0,15 s]; (b) [10 s, $15 \mathrm{~s}$; ; estimation of $f_{2}\left(\bar{x}_{2}\right)+d_{2}(t)$ by using TPPC in time period (c) $[0,15 \mathrm{~s}]$; (d) $[10 \mathrm{~s}, 15 \mathrm{~s}]$.

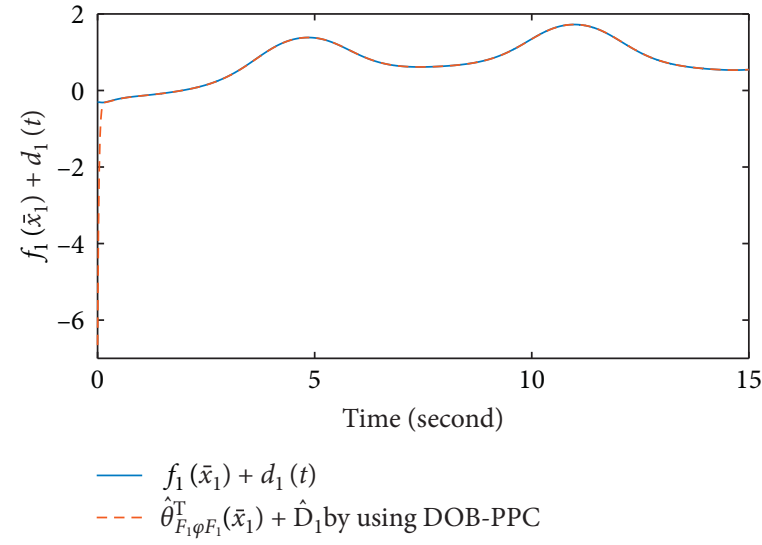

(a)

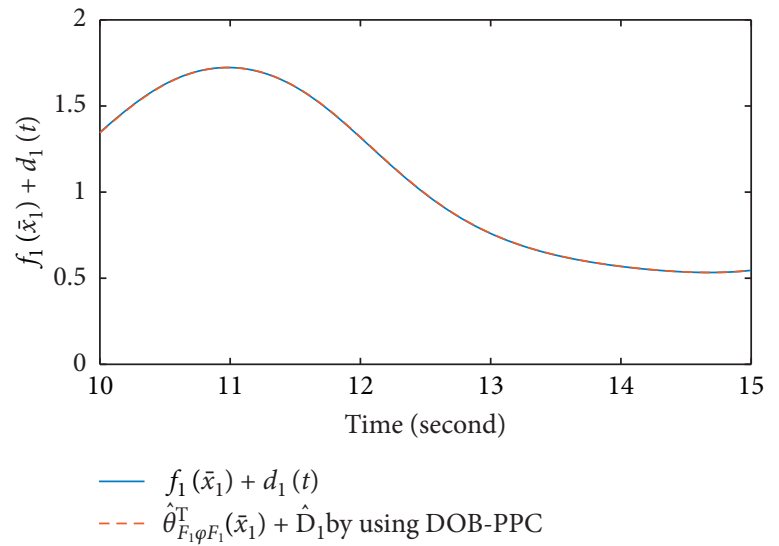

(b)

Figure 4: Continued. 


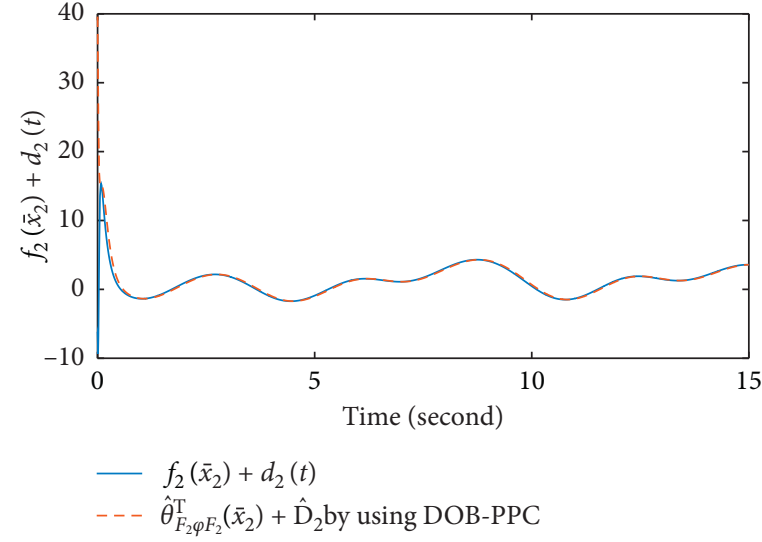

(c)

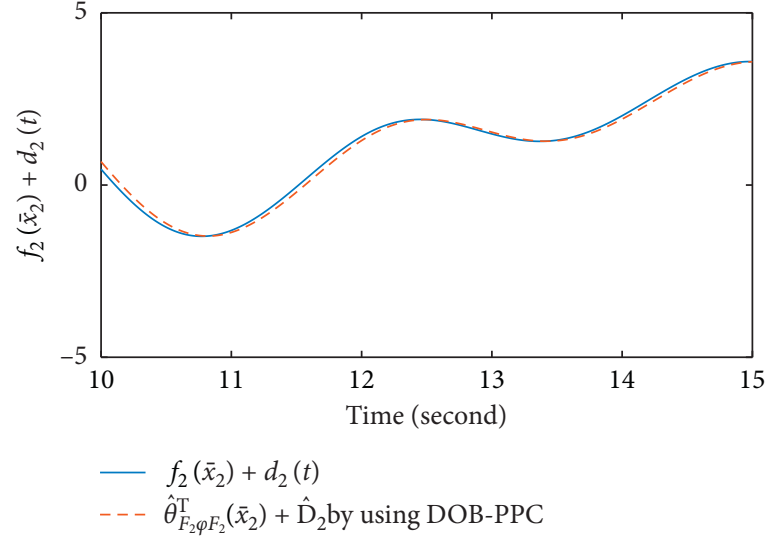

(d)

Figure 4: Estimation of $f_{1}\left(\bar{x}_{1}\right)+d_{1}(t)$ by using DOB-PPC in time period (a) [0,15 s]; (b) [10 s, $\left.15 \mathrm{~s}\right]$; estimation of $f_{2}\left(\bar{x}_{2}\right)+d_{2}(t)$ by using DOB-PPC in time period (c) $[0,15 \mathrm{~s}]$; (d) $[10 \mathrm{~s}, 15 \mathrm{~s}]$.

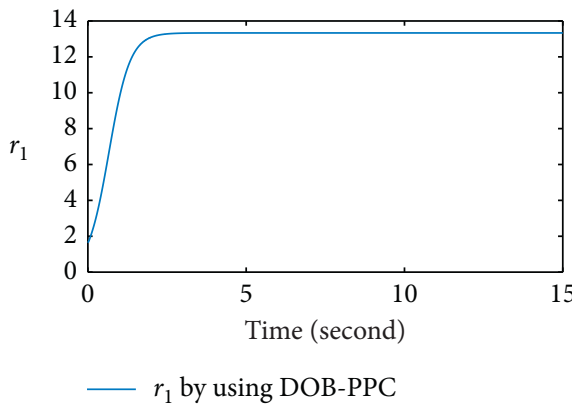

(a)

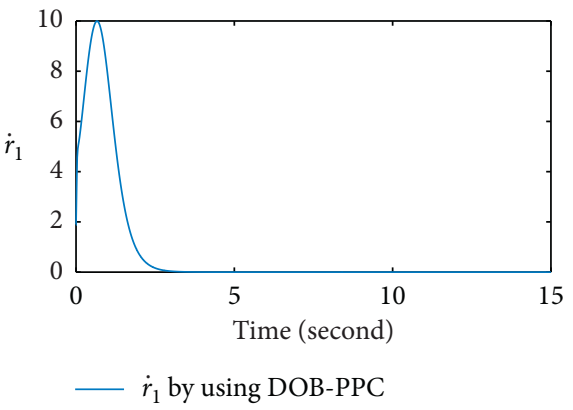

(b)

FIgURE 5: Time response of (a) $r_{1}$ and (b) $\dot{r}_{1}$.

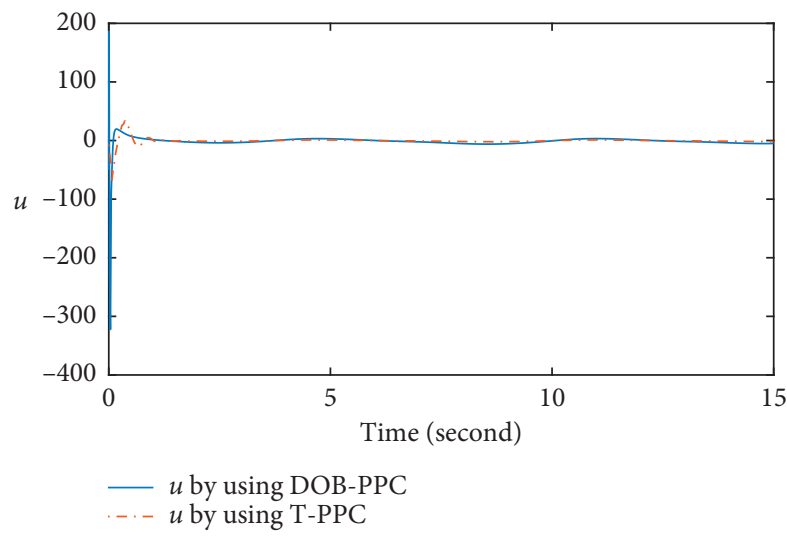

(a)

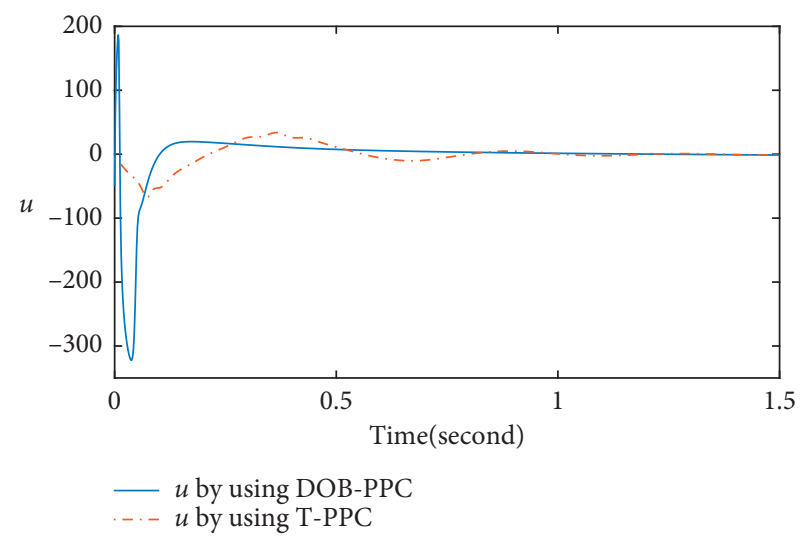

(b)

Figure 6: Time response of controller $u$ for two methods in time period (a) [0, $15 \mathrm{~s}]$; (b) [0, $1.5 \mathrm{~s}$.

confirms that the error effect of DOB-PPC is better than that of T-PPC.

The estimation of $f_{1}\left(\mathbf{x}_{1}\right)+d_{1}(t)$ and $f_{2}\left(\mathbf{x}_{2}\right)+d_{2}(t)$ for T-PPC and DOB-PPC is shown in Figures 3 and 4 . It can be found that the estimated effect of $f_{1}\left(\mathbf{x}_{1}\right)+d_{1}(t)$ and $f_{2}\left(\mathbf{x}_{2}\right)+d_{2}(t)$ is not satisfactory by using T-PPC, while this estimated effect is greatly improved by using DOB-PPC.

From (25), $\dot{r}_{1}$ appears in compact sets $\Omega_{z_{1}}, \Omega_{\widetilde{z}_{1}}$, and $\Omega_{\widetilde{D}_{1}}$. If $\dot{r}_{1}$ is infinite, this will cause the above compact sets to not exist. By observing Figure 5, we find that $r_{1}$ tends to a certain 
positive value, and $\dot{r}_{1}$ tends to zero, and this simulation result also confirms the rationality of $\left(z_{1}^{2} / 2 r_{1}\right)$ in Remark 3 . Finally, the control input of the two methods is shown in Figure 6. It can be seen from Figure 6 that the energy of DOB-PPC is greater than that of T-PPC at the beginning of the control process, and then the energy of the two control methods quickly approaches the same level. The results of the two methods are that the proposed DOB-PPC can significantly improve the tracking performance and the estimation accuracy of system uncertainties. So, it can be concluded that the proposed DOB-PPC is more effective than T-PPC.

\section{Conclusion}

In this paper, the composite prescribed performance control scheme is investigated for uncertain strict-feedback systems with unknown external disturbances. To estimate the unknown nonlinear functions $f_{i}\left(\overline{\mathbf{x}}_{i}\right)$ and unknown disturbances $d_{i}(t)$, RBFNNs and disturbance observers are employed. The observer $\widehat{D}_{i}$ constructed here does not estimate the external disturbance $d_{i}(t)$ but estimates the external disturbance $d_{i}(t)$ and approximates error $\varepsilon_{F_{i}}$. With the composite $\mathrm{NN}$ updating laws and disturbance observers, $f_{i}\left(\overline{\mathbf{x}}_{i}\right)+d_{i}(t)$ can be accurately estimated. Meanwhile, the whole closed-loop system can ensure stability by using PPC and backstepping technique. Moreover, the tracking error $e_{1}$ satisfies PPB (10). Finally, an example shows the effectiveness of the proposed method. Through the research in this paper, DOB-PPC with nonlinear control inputs will become the next research direction.

\section{Appendix}

By using RBFNNs,

$$
\bar{F}_{i}\left(\overline{\mathbf{x}}_{i}\right)=\vartheta_{\bar{F}_{i}}^{* T} \varphi_{\bar{F}_{i}}\left(\overline{\mathbf{x}}_{i}\right)+\varepsilon_{\bar{F}_{i}}, \quad i=1,2,
$$

where $\mathcal{E}_{\bar{F}_{i}}$ is bounded, i.e., there exists a positive constant $\delta_{i}^{*}$ such that $\left|\varepsilon_{\bar{F}_{i}}\right| \leq \delta_{i}^{*}$. So, we have

$$
\bar{F}_{i}\left(\overline{\mathbf{x}}_{i}\right)=\widetilde{\vartheta}_{\bar{F}_{i}}^{T} \varphi_{\bar{F}_{i}}\left(\overline{\mathbf{x}}_{i}\right)+\widehat{\vartheta}_{\overline{F_{i}}}^{T} \varphi_{\bar{F}_{i}}\left(\overline{\mathbf{x}}_{i}\right)+\varepsilon_{\bar{F}_{i}}\left(\overline{\mathbf{x}}_{i}\right),
$$

where $\widetilde{\vartheta}_{\bar{F}_{i}}=\vartheta_{\bar{F}_{i}}^{*}-\widehat{\vartheta}_{\bar{F}_{i}}$, and $\widehat{\vartheta}_{\bar{F}_{i}}$ is the estimation of $\vartheta_{\bar{F}_{i}}^{*}$. Therefore, second-order nonlinear system (47) can be rewritten as

$$
\left\{\begin{array}{l}
\dot{x}_{1}=\widehat{\vartheta} \frac{T}{F_{1}} \varphi_{\bar{F}_{1}}\left(\overline{\mathbf{x}}_{1}\right)+\widetilde{\mathfrak{\vartheta}} \frac{T}{F_{1}} \varphi_{\bar{F}_{1}}\left(\overline{\mathbf{x}}_{1}\right)+g_{1}\left(\overline{\mathbf{x}}_{1}\right) x_{2}+\varepsilon_{\bar{F}_{1}}\left(\overline{\mathbf{x}}_{1}\right), \\
\dot{x}_{2}=\widehat{\vartheta} \frac{T}{F_{2}} \varphi_{\bar{F}_{2}}\left(\overline{\mathbf{x}}_{2}\right)+\widetilde{\mathfrak{\vartheta}} \frac{T}{F_{2}} \varphi_{\bar{F}_{2}}\left(\overline{\mathbf{x}}_{2}\right)+g_{2}\left(\overline{\mathbf{x}}_{2}\right) u+\varepsilon_{\bar{F}_{2}}\left(\overline{\mathbf{x}}_{2}\right), \\
y=x_{1} .
\end{array}\right.
$$

For comparison with traditional PPB (1), we let $\lambda=1$, so the PPB of tracking error $e=x_{1}-x_{d}$ is set as

$$
-\mu(t)<e<\mu(t), \mu(t)=\left(\mu_{0}-\mu_{\infty}\right) e^{-\lambda_{1} t}+\mu_{\infty} .
$$

Define transformation variable $\bar{z}_{1}=\tanh (e / \mu)$. Similarly, we only need to prove $\bar{z}_{1}$ is bounded, which can guarantee that the tracking error $e$ satisfies PPB (A.4). The following two steps explore the effectiveness of control method (48).

Step 1: the time derivative of $\bar{z}_{1}$ becomes

$$
\dot{\bar{z}}_{1}=\bar{r}_{1}\left(\widetilde{\vartheta}_{\overline{F_{1}}}^{T} \varphi_{\bar{F}_{1}}\left(\overline{\mathbf{x}}_{1}\right)+\widehat{\vartheta}_{\bar{F}_{1}}^{T} \varphi_{\bar{F}_{1}}\left(\overline{\mathbf{x}}_{1}\right)+g_{1}\left(\overline{\mathbf{x}}_{1}\right) x_{2}+\varepsilon_{\bar{F}_{1}}\left(\overline{\mathbf{x}}_{1}\right)-\dot{x}_{d}+\bar{\Pi}\right),
$$

where $\quad \bar{r}_{1}=\left(1 / \mu\left(1-(e / \mu)^{2}\right)\right)>0$ and $\bar{\Pi}=-(e \dot{\mu} / \mu)$. Choose virtual control $\alpha_{1}$ as

$$
\alpha_{1}=\frac{-l_{1}^{-1} \widehat{\vartheta}_{\bar{F}_{1}}^{T} \varphi_{\bar{F}_{1}}\left(\overline{\mathbf{x}}_{1}\right)+\dot{x}_{d}-\bar{\Pi}-c_{1} \bar{z}_{1}}{g_{1}\left(\overline{\mathbf{x}}_{1}\right)},
$$

where $c_{1}$ is a positive design constant. Define $\bar{z}_{2}=x_{2}-\xi_{21}$, where $\xi_{21}$ is used to estimate $\alpha_{1}$ through the following second-order filter:

$$
\begin{aligned}
& \dot{\xi}_{21}=\xi_{22}, \\
& \dot{\xi}_{22}=\lambda_{21}^{2}\left[-\lambda_{22} \tanh \left(\xi_{21}-\alpha_{1}\right)-\lambda_{23} \tanh \left(\frac{\xi_{22}}{\lambda_{21}}\right)\right],
\end{aligned}
$$

where $\lambda_{21}, \lambda_{22}$, and $\lambda_{23}$ are design parameters. According to Lemma 1, when the appropriate parameters $\lambda_{21}, \lambda_{22}$, and $\lambda_{23}$ are selected, $\left|\xi_{21}-\alpha_{1}\right|$ is bounded. We assume this upper bound is $\bar{M}_{1}$. Define $e_{2}=\xi_{21}-\alpha_{1}$, and the parameter adaptive law for $\widehat{\vartheta}_{\bar{F}_{1}}$ is designed as

$$
\dot{\widehat{\vartheta}}_{\bar{F}_{1}}=\lambda_{F_{1}}\left(\bar{z}_{1} \varphi_{\bar{F}_{1}}\left(\overline{\mathbf{x}}_{1}\right)-\delta_{1} \widehat{\vartheta}_{\bar{F}_{1}}\right)
$$

where $\lambda_{F_{1}}$ and $\delta_{1}$ are positive design parameters.

Define the following Lyapunov function:

$$
V_{1}=\frac{1}{2 \bar{r}_{1}} \bar{z}_{1}^{2}+\frac{1}{2 \lambda_{F_{1}}} \widetilde{\vartheta}_{\bar{F}_{1}}^{T} \widetilde{\vartheta}_{\bar{F}_{1}} .
$$

According to (A.5)-(A.8) and $x_{2}=\bar{z}_{2}+e_{2}+\alpha_{1}$, the time derivative of $V_{1}$ is

$$
\begin{aligned}
\dot{V}_{1}= & -\left(c_{1}+\frac{\dot{\bar{r}}_{1}}{2 \bar{r}_{1}^{2}}\right) \bar{z}_{1}^{2}+\bar{z}_{1} g_{1}\left(\overline{\mathbf{x}}_{1}\right) \bar{z}_{2}+\bar{z}_{1} g_{1}\left(\overline{\mathbf{x}}_{1}\right) e_{2} \\
& +\bar{z}_{1} \varepsilon_{\bar{F}_{1}}\left(\overline{\mathbf{x}}_{1}\right)+\delta_{1} \widetilde{\vartheta}_{\bar{F}}^{T} \widehat{\vartheta}_{1}^{T} \bar{F}_{1} .
\end{aligned}
$$

If $z_{2}$ is bounded, i.e., there exists a positive constant $\bar{K}_{2}$ such that $\left|\bar{z}_{2}\right| \leq \bar{K}_{2}$, and according to Young's inequality, we have 


$$
\begin{aligned}
\bar{z}_{1} e_{2} g_{1}\left(\overline{\mathbf{x}}_{1}\right) \leq \frac{\bar{z}_{1}^{2}}{2}+\frac{\bar{g}_{1} \bar{M}_{1}^{2}}{2}, \\
\bar{z}_{1} g_{1}\left(\overline{\mathbf{x}}_{1}\right) \bar{z}_{2} \leq \frac{\bar{z}_{1}^{2}}{2}+\frac{\bar{g}_{1}^{2} \bar{K}_{2}^{2}}{2}, \\
\delta_{1} \widetilde{\vartheta}_{\bar{F}_{1}}^{T} \widehat{\vartheta}_{\bar{F}_{1}} \leq-\frac{\delta_{1}}{2}\left\|\widetilde{\vartheta}_{\bar{F}_{1}}\right\|^{2}+\frac{\delta_{1}}{2}\left\|\vartheta_{\bar{F}_{1}}^{*}\right\|^{2}, \\
\bar{z}_{1} \varepsilon_{\bar{F}_{1}}\left(\overline{\mathbf{x}}_{1}\right) \leq \frac{\bar{z}_{1}^{2}}{2}+\frac{\delta_{1}^{* 2}}{2} .
\end{aligned}
$$

Substituting (A.11) into (A.10), we obtain

$$
\dot{V}_{1} \leq-\left(c_{1}-\frac{3}{2}+\frac{\dot{\bar{r}}_{1}}{2 \bar{r}_{1}^{2}}\right) \bar{z}_{1}^{2}-\frac{\delta_{1}}{2}\left\|\widetilde{\vartheta}_{\bar{F}_{1}}\right\|^{2}+\bar{R}_{1},
$$

where $\quad \bar{R}_{1}=\left(\bar{g}_{1} \bar{M}_{1}^{2} / 2\right)+\left(\bar{g}_{1}^{2} \bar{K}_{2}^{2} / 2\right)+\left(\delta_{1} / 2\right)\left\|9_{\bar{F}_{1}}^{*}\right\|^{2}+$ $\left(\delta_{1}^{* 2} / 2\right)$. Let $c_{1}>(3 / 2)-\left(\dot{\bar{r}}_{1} / 2 \bar{r}_{1}^{2}\right)$, and define the following compact sets:

$$
\begin{aligned}
& \Omega_{\bar{z}_{1}}=\left\{\bar{z}_{1}|| \bar{z}_{1} \mid \leq \sqrt{\frac{\bar{R}_{1}}{c_{1}-(3 / 2)+\left(\dot{\bar{r}}_{1} / 2 \bar{r}_{1}^{2}\right)}}\right\}, \\
& \Omega_{\widetilde{\vartheta}_{\bar{F}_{1}}}=\left\{\widetilde{\vartheta}_{\bar{F}_{1}}\left|\widetilde{\vartheta}_{\bar{F}_{1}}\right| \leq \sqrt{\frac{\bar{R}_{1}}{\left(\delta_{1} / 2\right)}}\right\} .
\end{aligned}
$$

It can be seen that $\bar{z}_{1} \notin \Omega_{z_{1}}$ or $\widetilde{\vartheta}_{\bar{F}_{1}} \notin \Omega_{\widetilde{\vartheta}_{\bar{F}_{1}}}$, and we have $\dot{V}_{1}<0$. Thus, $z_{1}$ and $\widetilde{\vartheta}_{\bar{F}_{1}}$ are all semiglobally uniformly bounded. The above conclusion is based on the fact that the variable $\bar{z}_{2}$ is bounded. Now, we need to prove that $\bar{z}_{2}$ is bounded.

Step 2:

The time derivative of $\bar{z}_{2}$ produces

$\dot{\bar{z}}_{2}=\widehat{\vartheta}_{\bar{F}_{2}}^{T} \varphi_{\bar{F}_{2}}\left(\overline{\mathbf{x}}_{2}\right)+\widetilde{\vartheta}_{\bar{F}_{2}}^{T} \varphi_{\bar{F}_{2}}\left(\overline{\mathbf{x}}_{2}\right)+g_{2}\left(\overline{\mathbf{x}}_{2}\right) u+\varepsilon_{\bar{F}_{2}}\left(\overline{\mathbf{x}}_{2}\right)-\xi_{22}$.

The control $u$ is designed as

$$
u=\frac{-\widehat{\vartheta}_{\bar{F}_{2}}^{T} \varphi_{\bar{F}_{2}}\left(\overline{\mathbf{x}}_{2}\right)+\xi_{22}-c_{2} \bar{z}_{2}}{g_{2}\left(\overline{\mathbf{x}}_{2}\right)},
$$

where $c_{2}$ is a positive constant. And the parameter adaptive law for $\widehat{\vartheta}_{\bar{F}_{2}}$ is designed as

$$
\dot{\hat{\vartheta}}_{\bar{F}_{2}}=\lambda_{F_{2}}\left[\bar{z}_{2} \varphi_{\bar{F}_{2}}\left(\overline{\mathbf{x}}_{2}\right)-\delta_{2} \widehat{\vartheta}_{\bar{F}_{2}}\right] \text {, }
$$

where $\lambda_{F_{2}}$ and $\delta_{2}$ are positive design parameters.

Consider the following Lyapunov function:

$$
V_{2}=\frac{1}{2}\left(\bar{z}_{2}^{2}+\frac{1}{\lambda_{F_{2}}} \widetilde{\vartheta}_{\bar{F}_{2}}^{T} \widetilde{\vartheta}_{\bar{F}_{2}}\right) \text {. }
$$

According to (A.15) and (A.16), the time derivative of $V_{2}$ is

$$
\dot{V}_{2}=-c_{2} \bar{z}_{2}^{2}-\bar{z}_{2} \varepsilon_{\bar{F}_{2}}\left(\overline{\mathbf{x}}_{2}\right)+\delta_{2} \widetilde{\vartheta}_{\bar{F}_{2}}^{T} \widehat{\vartheta}_{\bar{F}_{2}}^{T}
$$

Consider the following facts:

$$
\begin{aligned}
-\bar{z}_{2} \varepsilon_{\bar{F}_{2}}\left(\overline{\mathbf{x}}_{2}\right) & \leq \frac{\bar{z}_{2}^{2}}{2}+\frac{\delta_{2}^{* 2}}{2}, \\
\delta_{2} \widetilde{\vartheta}_{\bar{F}_{2}}^{T} \widehat{\vartheta}_{\bar{F}_{2}} & \leq-\frac{\delta_{2}}{2}\left\|\widetilde{\vartheta}_{\bar{F}_{2}}\right\|^{2}+\frac{\delta_{2}}{2}\left\|\vartheta_{\bar{F}_{2}}^{*}\right\|^{2} .
\end{aligned}
$$

Substituting (A.19) into (A.18), we obtain

$$
\dot{V}_{2} \leq-\left(c_{2}-\frac{1}{2}\right) \bar{z}_{2}^{2}-\frac{\delta_{2}}{2}\left\|\widetilde{\vartheta}_{\bar{F}_{2}}\right\|^{2}+\bar{R}_{2}
$$

where $\bar{R}_{2}=\left(\delta_{2}^{* 2} / 2\right)+\left(\delta_{2} / 2\right)\left\|\vartheta_{\bar{F}_{2}}^{*}\right\|^{2}$. Let $c_{2}>(1 / 2)$, and define the following compact sets:

$$
\begin{aligned}
& \Omega_{\bar{z}_{2}}=\left\{\bar{z}_{2}|| \bar{z}_{2} \mid \leq \sqrt{\frac{\bar{R}_{2}}{c_{2}-(1 / 2)}}\right\}, \\
& \Omega_{\widetilde{\vartheta}_{\bar{F}_{2}}}=\left\{\widetilde{\vartheta}_{\bar{F}_{n}}\left|\widetilde{\vartheta}_{\bar{F}_{n}}\right| \leq \sqrt{\frac{\bar{R}_{2}}{\left(\delta_{2} / 2\right)}}\right\} .
\end{aligned}
$$

Obviously, $\bar{z}_{2} \notin \Omega_{\bar{z}_{2}}$ or $\widetilde{\vartheta}_{\bar{F}_{2}} \notin \Omega_{\widetilde{\vartheta}_{\bar{F}_{2}}}$, and we have $\dot{V}_{2}<0$. Thus, $\bar{z}_{2}$ and $\widetilde{\vartheta}_{\bar{F}_{2}}$ are semiglobally uniformly bounded. So, the controller $u$ can guarantee that $\bar{z}_{2}$ is bounded, and the boundedness of $\bar{z}_{2}$ guarantees that $\bar{z}_{1}$ is bounded. Therefore, the tracking error $e$ satisfies PPB (A.4). The above discussion proves the controllability of method (48).

\section{Data Availability}

All datasets generated for this study are included in the manuscript.

\section{Conflicts of Interest}

The authors declare that there are no conflicts of interest regarding the publication of this paper.

\section{Acknowledgments}

This work was supported in part by the Anhui Natural Science Foundation under Grant nos. 1608085QA05 and 2008085MF200, the Anhui Province Excellent Talents Project under Grant no. gxyq2018065, the Natural Science Research Projects in Anhui Universities under Grant nos. KJ2019ZD48 and KJ2019A0695, and the Innovative Research Team in Huainan Normal University under Grant no. XJTD202008.

\section{References}

[1] J. A. Farrell, M. Polycarpou, M. Sharma, and W. Wenjie Dong, "Command filtered backstepping," IEEE Transactions on Automatic Control, vol. 54, no. 6, pp. 1391-1395, 2009.

[2] E. Wei, T. Li, J. Li, Y. Hu, and Q. Li, "Neural network-based adaptive dynamic surface control for inverted pendulum system," in Foundations and Practical Applications of 
Cognitive Systems and Information Processing, pp. 695-704, Springer, Berlin, Germany, 2014.

[3] Y. Guo and J. Liu, "Neural network based adaptive dynamic surface control for flight path angle," in Proceedings of the 2012 IEEE 51st IEEE Conference on Decision and Control (CDC), pp. 5374-5379, IEEE, Maui, HI, USA, December 2012.

[4] D. Wang and J. Huang, "Neural network-based adaptive dynamic surface control for a class of uncertain nonlinear systems in strict-feedback form," IEEE Transactions on Neural Networks, vol. 16, no. 1, pp. 195-202, 2005.

[5] S. Ri, J. Huang, Y. Wang, C. Tao, J. Kim, and K. Pak, "Dynamic surface control of mobile wheeled inverted pendulum systems with nonlinear disturbance observer," IFAC Proceedings Volumes, vol. 47, no. 3, pp. 4505-4510, 2014.

[6] J. Yu, P. Shi, W. Dong, B. Chen, and C. Lin, "Neural networkbased adaptive dynamic surface control for permanent magnet synchronous motors," IEEE Transactions on Neural Networks and Learning Systems, vol. 26, no. 3, pp. 640-645, 2014.

[7] O. Aung, Y. Lin, and Q. Zhao, "Fuzzy based adaptive dynamic surface control for a class of uncertain nonlinear systems in strict-feedback form," in Proceedings of the 2009 9th International Conference on Electronic Measurement \& Instruments, pp. 3-499, IEEE, Beijing, China, August 2009.

[8] A. Levant, "Higher-order sliding modes, differentiation and output-feedback control," International Journal of Control, vol. 76, no. 9-10, pp. 924-941, 2003.

[9] J. Yu, L. Zhao, H. Yu, C. Lin, and W. Dong, "Fuzzy finite-time command filtered control of nonlinear systems with input saturation," IEEE Transactions on Cybernetics, vol. 48, no. 8, pp. 2378-2387, 2017.

[10] Y. Pan and H. Yu, "Composite learning robot control with guaranteed parameter convergence," Automatica, vol. 89, pp. 398-406, 2018.

[11] Y. Pan, T. Sun, Y. Liu, and H. Yu, "Composite learning from adaptive backstepping neural network control," Neural Networks, vol. 95, pp. 134-142, 2017.

[12] H. Liu, Y. Pan, and J. Cao, "Composite learning adaptive dynamic surface control of fractional-order nonlinear systems," IEEE Transactions on Cybernetics, vol. 50, no. 6, p. 2557, 2020.

[13] H. Liu, H. Wang, J. Cao, A. Alsaedi, and T. Hayat, "Composite learning adaptive sliding mode control of fractional-order nonlinear systems with actuator faults," Journal of the Franklin Institute, vol. 356, no. 16, pp. 9580-9599, 2019.

[14] H. Li, J. Wang, H.-K. Lam, Q. Zhou, and H. Du, "Adaptive sliding mode control for interval type-2 fuzzy systems," IEEE Transactions on Systems, Man, and Cybernetics: Systems, vol. 46, no. 12, pp. 1654-1663, 2016.

[15] Y. Li, S. Sui, and S. Tong, "Adaptive fuzzy control design for stochastic nonlinear switched systems with arbitrary switchings and unmodeled dynamics," IEEE Transactions on Cybernetics, vol. 47, no. 2, pp. 403-414, 2016.

[16] Y.-J. Liu, M. Gong, S. Tong, C. L. P. Chen, and D.-J. Li, "Adaptive fuzzy output feedback control for a class of nonlinear systems with full state constraints," IEEE Transactions on Fuzzy Systems, vol. 26, no. 5, pp. 2607-2617, 2018.

[17] H. Liu, Y. Pan, J. Cao, Y. Zhou, and H. Wang, "Positivity and stability analysis for fractional-order delayed systems: a T-S fuzzy model approach," IEEE Transactions on Fuzzy Systems, p. 1, 2020.

[18] S. Vrkalovic, T.-A. Teban, and I.-D. Borlea, "Stable takagisugeno fuzzy control designed by optimization," International Journal of Artificial Intelligence, vol. 15, no. 2, pp. 17-29, 2017.
[19] R.-E. Precup, R.-C. David, and E. M. Petriu, "Grey wolf optimizer algorithm-based tuning of fuzzy control systems with reduced parametric sensitivity," IEEE Transactions on Industrial Electronics, vol. 64, no. 1, pp. 527-534, 2016.

[20] M. Chen, P. Shi, and C.-C. Lim, "Adaptive neural fault-tolerant control of a 3-dof model helicopter system," IEEE Transactions on Systems, Man, and Cybernetics: Systems, vol. 46, no. 2, pp. 260-270, 2015.

[21] B. Xu, "Composite learning control of flexible-link manipulator using NN and DOB," IEEE Transactions on Systems, Man, and Cybernetics: Systems, vol. 48, no. 11, pp. 1979-1985, 2017.

[22] B. Xu, Z. Shi, and C. Yang, "Composite fuzzy control of a class of uncertain nonlinear systems with disturbance observer," Nonlinear Dynamics, vol. 80, no. 1-2, pp. 341-351, 2015.

[23] B. Xu, F. Sun, Y. Pan, and B. Chen, "Disturbance observer based composite learning fuzzy control of nonlinear systems with unknown dead zone," IEEE Transactions on Systems, Man, and Cybernetics: Systems, vol. 47, no. 8, pp. 1854-1862, 2016.

[24] S. Mobayen and S. Javadi, "Disturbance observer and finitetime tracker design of disturbed third-order nonholonomic systems using terminal sliding mode," Journal of Vibration and Control, vol. 23, no. 2, pp. 181-189, 2017.

[25] M. R. Mokhtari, B. Cherki, and A. C. Braham, "Disturbance observer based hierarchical control of coaxial-rotor UAV," ISA Transactions, vol. 67, pp. 466-475, 2017.

[26] H. Shim, G. Park, Y. Joo, J. Back, and N. H. Jo, "Yet another tutorial of disturbance observer: robust stabilization and recovery of nominal performance," Control Theory and Technology, vol. 14, no. 3, pp. 237-249, 2016.

[27] S. B. Phadke, P. Shendge, and V. S. Wanaskar, "Control of antilock braking systems using disturbance observer with a novel nonlinear sliding surface," IEEE Transactions on Industrial Electronics, vol. 67, no. 8, pp. 6815-6823, 2019.

[28] M. Chen, Q. Wu, C. Jiang, and B. Jiang, "Guaranteed transient performance based control with input saturation for near space vehicles," Science China Information Sciences, vol. 57, no. 5, pp. 1-12, 2014.

[29] H. Liu, S. Li, J. Cao, G. Li, A. Alsaedi, and F. E. Alsaadi, "Adaptive fuzzy prescribed performance controller design for a class of uncertain fractional-order nonlinear systems with external disturbances," Neurocomputing, vol. 219, pp. 422430, 2017.

[30] C. P. Bechlioulis and G. A. Rovithakis, "Robust adaptive control of feedback linearizable MIMO nonlinear systems with prescribed performance," IEEE Transactions on Automatic Control, vol. 53, no. 9, pp. 2090-2099, 2008.

[31] X. Bu, G. He, and D. Wei, "A new prescribed performance control approach for uncertain nonlinear dynamic systems via back-stepping," Journal of the Franklin Institute, vol. 355, no. 17, pp. 8510-8536, 2018. 